\title{
Heavy Flavor and B Physics
}

\author{
George W.S. Hou \\ Department of Physics, National Taiwan University, Taipei, Taiwan 10617, R.O.C. \\ wshou@phys.ntu.edu.tw
}

This note is based on several lectures on B physics, given to unspecialized students in particle physics. The physics of flavor is presented from an experimental point of view. The theme is CPV measurement and the CKM matrix. Some recent topics are also discussed, with a projection into the future.

\section{Setting the Scene}

With the riddle of the nucleus resolved by the neutron discovery and the pion proposal, isospin symmetry, or $m_{p} \cong m_{n}$, was an early harbinger of "flavor", which is now understood as due to $m_{u}, m_{d} \ll m_{N}$. The $\pi \rightarrow \mu \rightarrow e$ decay chain, discovered in 1947 using emulsions developed during WWII, gave us the muon, which is "just like the electron", but 200 times heavier. I.I. Rabi's question: "Who ordered that?", marks the beginning of flavor physics.

The muon is the template of 3-body tree level weak decay $\left(x=m_{e}^{2} / m_{\mu}^{2}\right)$,

$$
\Gamma_{\mu}=\frac{G_{F}^{2} m_{\mu}^{5}}{192 \pi^{3}}\left(1-8 x+8 x^{3}-x^{4}-12 x^{2} \ln x\right) .
$$

Loop induced $\mu \rightarrow e \gamma$ and $g-2$ processes are still of great interest.

\subsection{Kaon: the "Strange" Template}

Repeating the muon discovery, "strangeness" became the template for flavor.

Long Lifetime With $m_{K}^{2} / m_{\pi}^{2} \sim 13$ large, it was hard to understand the smallness of $\Gamma_{K} / \Gamma_{\pi} \sim 2$. This "strangeness" lead to the Cabibbo proposal. In quark language it states that one has $d_{C}=d \cos \theta_{C}+s \sin \theta_{C}$ in weak interactions, with the Cabibbo angle $\sin \theta_{C} \simeq 0.22$ suppressing strangeness decay. 
$K^{0}-\bar{K}^{0}$ Mixing With strangeness, $K^{0}$ and $\bar{K}^{0}$ are antiparticles of each other, and eigenstates of the strong interaction, but can mix via the weak interaction into the $C P$ even and odd $K_{1}^{0}$ and $K_{2}^{0}$ mesons, where $C$ and $P$ are charge conjugation and parity operations. With parity violation, it was believed that $C P$ remained conserved, then $K_{1} \rightarrow \pi \pi$ and $K_{2} \rightarrow \pi \pi \pi$ but not vice versa. With $3 m_{\pi} \sim m_{K}$, one gets $\tau_{K_{1}} \ll \tau_{K_{2}}$, agreeing with data, and one observes the so-called mixing, or oscillation in time, of $K^{0} \leftrightarrow \bar{K}^{0}$.

Suppressed SCNC Neutral current induced $s \rightarrow d$ effects were expected at $\sim \sin \theta_{C} \cos \theta_{C}$ of charged current effects. One then can not understand the slowness of $K^{0}-\bar{K}^{0}$ oscillations, while $K_{2} \rightarrow \mu^{+} \mu^{-}$was also highly suppressed. Glashow, Iliopoulos and Maiani (GIM) proposed adding a second weak doublet $s_{C}=-d \sin \theta_{C}+s \cos \theta_{C}$ to the Cabibbo proposal. This completes a $2 \times 2$ rotation in charge $-1 / 3$ flavor space. Miraculously, this not only removes tree level Strangeness Changing Neutral Currents (SCNS), it renders the second order weak box diagram calculable, with both $u$ and $c$ (partner of $s$ in the new doublet) quarks in the loop, allowing the charm mass to be estimated.

$C P$ Violation In 1964 it was discovered that the " $K_{2}^{0}$ " meson actually decayed to $\pi \pi$ at $\sim 1 / 500$, which can be parameterized by an admixture of $\left|\varepsilon_{K}\right| \sim$ $2 \times 10^{-3}$ of $K_{1}$ in " $K_{2}$ ". The mass eigenstates were therefore called $K_{S}$ and $K_{L}$, for short and long lived, respectively. CPV occurred in $K^{0}-\bar{K}^{0}$ mixing.

\section{Digression on CPV}

The invention of the GIM mechanism, together with the challenge to understand CPV, lead to a further observation. Kobayashi and Maskawa pointed out that, by enlarging the $2 \times 2$ rotation matrix to a $3 \times 3$ unitary matrix, the gauge theory of weak interactions has a unique, irremovable phase that is $C P$ violating. CPV called for the existence of 3 generations.

$C$ maps particles to their antiparticles. Combining with $P$, the $C P$ operation maps left-handed fermions to right-handed antifermions. Given that particle and antiparticle are on equal footing in dynamical theories such as QED (and QCD), it was hard to understand why the Universe seems to be made only of "particles", $p, n$ and $e$. Where have all the antimatter gone? To address this pressing question, and motivated by the discovery of CPV, Sakharov formulated the 3 conditions for the Matter Universe, i.e.

1) baryon number violation, 2) CPV, and 3) out of equilibrium.

However, the CPV observed in $\varepsilon_{K}$ within the KM model, is orders of magnitude too small. This adds to the importance of continued search for CPV.

It is illuminating to consider $\mathrm{CPV}$ in the quantum mechanical context. The essence of quantum, or wave, behavior is the interference of complex amplitudes, with a common $i$ in the time evolution of quantum states. From atomic to condensed matter physics, the dynamics is nothing but Maxwell theory, and the "reality" of the coupling constant $e$ is taken for granted. We now understand that gauge charge is real. 
Complexity in dynamics, the existence of complex couplings, such as the Yukawa couplings in the Standard Model (SM), makes quantum interference much more interesting — which is CPV phenomena.

\subsection{Heavy Flavor: $c / \tau / b / t$}

The story of heavy flavor starts with the discovery of charm in 1974, and culminates in the top discovery in 1995 that completed the 3rd generation.

On the opposite end, the neutrinos were recently found to have mass [1], but not more than eV scale (and probably far less). In between we have the familiar $u / d / s$ quarks and $e, \mu$ charged leptons. With QM underlying the periodic table, and QCD underlying the proliferation of hadrons, we do not yet know whether anything underlies the "spectroscopy of flavor".

\section{Charm}

The GIM mechanism required the existence of the "charm" quark, even an estimate of $m_{c}$ from $K^{0}-\bar{K}^{0}$ mixing. But this does not take away any glory from the experimental discovery of charm, first in its hidden form as $J / \psi$.

The discovery of $J$ in $p+\mathrm{Be} \rightarrow e^{+} e^{-}+X$ at BNL, and $\psi$ in $e^{+} e^{-} \rightarrow$ hadrons and leptons at SLAC, caused the "November Revolution": a new very heavy hadron with very narrow width. The narrowness can be qualitatively accounted for by invoking the OZI rule, which implied a new "flavor" quantum number beyond strangeness. The $J / \psi$ discovery precipitated the acceptance of quarks, acceptance of QCD as the underlying strong dynamics, and the existence of a new spectroscopy. The Standard Model became adopted.

The SPEAR $e^{+} e^{-}$collider with the solenoidal MARK I detector marked the start of $e^{+} e^{-}$collider physics. The $D$ mesons, or "open charm" ( $c \bar{q}$ mesons) as compared to the "hidden charm" ( $c \bar{c}$ mesons called charmonium), followed in 1975 . The resonance $\psi(3770)$, above $D \bar{D}$ threshold, decayed dominantly into $D \bar{D}$ meson pairs without OZI violation. Spectroscopic activities have been renewed recently, after a 25 year hiatus, by the discovery of new narrow states such as the $X(3872)[2]$ and $D_{s J}(2317)[3]$.

$D^{*}$ mesons, analogues of the $\rho$ and $K^{*}$ mesons, further confirmed the quark model picture, with $D^{* 0} \rightarrow D^{0} \pi^{0}, D^{0} \gamma$ while $D^{*+} \rightarrow D^{0} \pi^{+}, D^{+} \pi^{0}, D^{+} \gamma$ understood from kinematics. The pseudoscalar mesons, the lightest charmed particles, can decay only weakly. It was found that $\tau_{D^{+}} / \tau_{D^{0}} \sim 2.5$, indicating that hadronic effects are important for $D$ meson weak decay.

We shall not dwell much on charm physics, except a few special issues.

\section{Tau}

Although the KM picture for CPV anticipated the existence of the 3rd generation, there is no prediction for their masses. The discovery of the $\tau$ lepton 
followed swiftly after charm, however, in 1975 at SLAC, with the help of theoretical calculations that generalized from Eq. (1). From $\tau \rightarrow e \nu \bar{\nu}$ and $\mu \nu \bar{\nu}$ decay, one has the signature of $e^{+} e^{-} \rightarrow \tau^{+} \tau^{-} \rightarrow e^{ \pm} \mu^{\mp}+$ missing energy. The process $e^{+} e^{-} \rightarrow \tau^{+} \tau^{-}$has been studied from SPEAR up to LEP energies, confirming SM couplings of the $\tau$ lepton to great precision.

Again, we shall not cover tau physics except a couple of rare decays.

\section{Beauty}

The discovery of the bottom quark, or "beauty" flavor, repeated the $J / \psi$ : the hidden beauty $(b \bar{b}) \Upsilon$ meson was discovered in 1977 at Fermilab as a bump in $m_{\mu \mu}$ in $p+\mathrm{Be} \rightarrow \mu^{+} \mu^{-}+X$ events. This stimulated the development of the $e^{+} e^{-}$colliders CESR at Cornell, and DORIS at DESY, capable of running around $10 \mathrm{GeV}$ center of mass (CM) energies. By 1980, the CLEO detector operating at CESR found the $\Upsilon(4 S)$ resonance, which opened the way for open beauty meson studies. The $B$ mesons $(b \bar{q})$ were discovered in 1983, while the "bottomonium" spectroscopy further confirms the quark model and the underlying QCD. With $\tau_{B^{+}} / \tau_{B^{0}} \sim 1.076$, hadronic effects are far less important for $B$ mesons than for $D$ mesons.

\section{Top}

With $\tau$ and $b$ discovered, the charge $+2 / 3$ quark seemed just around the corner. But the top quark did not appear until 1995. At $174 \mathrm{GeV}$, it is uniquely heavy among fermions. Because of this, the top quark as a probe of flavor has yet to start, so we shall also not cover it.

\subsection{The Centrality of $B$}

The $c / \tau / t$ lifetimes are unsuppressed, hence loop induced decays are much more subdued. In contrast, $B$ physics is central to flavor and CPV, because it repeats the pattern seen in strangeness, and is in fact far richer.

We retrace the discoveries, highlight the physics, and point out the techniques that became crucial for $e^{+} e^{-}$and hadronic "B factories".

\section{Long $B$ Lifetime and CKM Pattern}

Vertex detectors with sub-mm accuracy, aimed at $c / \tau$ lifetime measurement, discovered the long $\tau_{b}$ in 1983. The MAC and MARK II experiments [4] at PEP observed large impact parameter events (high $p_{T}$ leptons with respect to the jet axis), implying that $b$ hadron lifetime was at ps level, several hundred times longer than scaling up from weak decay of the $\tau$. This implied that $V_{c b}$ and $V_{u b}$ are much smaller than $\lambda \equiv V_{u s} \sim 0.22$ and was not anticipated.

Reports in summer 1983 on semileptonic $b$ decay indicated [5] $b \rightarrow c \ell \nu$ dominance, i.e. the $E_{\ell}$ spectrum cuts off with a recoil system compatible with 
the $D$ meson, but no higher $E_{\ell}$ component corresponding to $b \rightarrow u \ell \nu$. This was the first clear indication that $\left|V_{u b}\right| \ll\left|V_{c b}\right|$. We now know that [6]

$$
V_{u s} \equiv \lambda \sim 0.22, V_{c b} \equiv A \lambda^{2} \simeq 0.04,\left|V_{u b}\right| \equiv A \lambda^{3} \sqrt{\rho^{2}+\eta^{2}} \sim 0.004
$$

\section{Large $B^{0}$ Mixing and Heavy Top}

"Accompanying" the SN1987A supernova, the ARGUS experiment announced the discovery of rather large $B^{0}-\bar{B}^{0}$ mixing [7]. Like sign dilepton events with $\sim 100 \mathrm{pb}^{-1}$ data at the DORIS storage ring running on the $\Upsilon(4 S)$ gave the mixing parameter $r=0.21 \pm 0.08$, just below a preceding limit given in 1986 by the CLEO experiment, and the confirmation from CLEO came in 1989. The $x_{B}=\Delta m_{B} / \Gamma_{B} \sim 1$ value is in contrast to the prejudice that $x_{B} \ll x_{K}$, which was due to the prevailing expectation, since 1977, that the top quark was "just around the corner". A flourish of papers followed to claim a heavy top, $m_{t}>50 \mathrm{GeV}$. It took another decade for the top quark to appear.

\section{Rare $B$ Decays: $B \rightarrow K^{*} \gamma$ and $X_{s} \gamma$}

In 1993, the CLEO experiment observed $13 B \rightarrow K^{*} \gamma$ events with roughly 1.4 $\mathrm{fb}^{-1}$ data, and measured the radiative decay at $4-5 \times 10^{-5}$ [8]. This was the first evidence for $b \rightarrow s \gamma$, forbidden within the SM at the tree level by gauge invariance. The measurement came after a major investment on $\mathrm{CsI}(\mathrm{T} \ell)$ crystal electromagnetic calorimeter (ECAL). By end of 1994, CLEO observed [8] the inclusive $b \rightarrow s \gamma$ process, by partially reconstructing $X_{s}$ via $K+n \pi$, with $n$ up to 4 , and at most one $\pi^{0}$. The $B \rightarrow X_{s} \gamma$ rate, identified with $b \rightarrow s \gamma$, was found to be $2-3 \times 10^{-4}$, in agreement with SM expectations enhanced by QCD. The $m_{X_{s}}$ spectrum from partial reconstruction shows a peak consistent with $K^{*}$ at low mass, and resonances at higher mass.

The rare $b \rightarrow s \gamma$ decay is mediated by "penguin" loops, with real photon emission. The $b$ quark first "decays" to a virtual $W$ and $t$ pair. With the emission of the photon, the virtual $W t$ pair recombines into an $s$ quark. The photon couples to the $b \rightarrow s$ transition current via an effective $\sigma_{\mu \nu}$ dipole, and at leading order in strong interactions, the process is highly GIM suppressed. But, when one considers QCD corrections, the GIM suppression is circumvented at next order [9]. Thus, though higher order, because of extreme suppression of the leading term, the rate becomes greatly enhanced at $\alpha_{s}$ order. The CLEO measurement confirms this picture. One outcome of this measurement is the most stringent bound on the charged Higgs boson mass, $m_{H^{+}}>320 \mathrm{GeV}$ [6], by replacing $W^{+}$by $H^{+}$in the loop [10].

With the improved CLEO II, the ARGUS experiment came to an end.

\section{Towards B Factories}

With long $B$ lifetime, large $B^{0}-\bar{B}^{0}$ mixing and rare "BCNC" decays, one anticipates further "repeat" of CPV phenomena in the B system. It turned out to 
be far richer than the kaon system. The mixing-decay interference mechanism was proposed (Lecture 2), which received great boost from the observation of large $B$ mixing in 1987. Before long, there was a world competition for "B factories", to observe CPV in B system.

The KEK and SLAC proposals picked up the idea [11] of an asymmetric $e^{+} e^{-}$collider. With a boosted $B$ meson, new silicon vertex detectors allowed one access to mixing-decay interference measurement. Construction of KEKB and PEP-II started in the first half of the 1990s.

In 1992, the CDF experiment at the Tevatron fully reconstructed $\sim 10$ $B^{ \pm} \rightarrow J / \psi K^{ \pm}$events [12], demonstrating that B physics at hadron colliders is feasible. With the advantage in cross section, and techniques such as vertexing, forward detector concepts were advanced to further utilize a larger boost and allow more space (especially for particle identification, or PID, and ECAL). The LHCb detector will run in 2008 at LHC. Unfortunately, the BTeV detector, proposed for the Tevatron, was canceled in 2005.

\subsection{The B Factories}

The KEKB accelerator, operating with $3.5 e^{+}$on $8.5 \mathrm{GeV} e^{-}$, produces the $\Upsilon(4 S)$ resonance with $\beta \gamma=0.425$, while PEP-II has $\beta \gamma=0.56$. The main difference between the two is in beam crossing at the Interaction Region (IR). KEKB uses finite angle crossing, while PEP-II uses the more conservative zero angle crossing, with beam separation by permanent magnets at IR.

PEP-II took the initial lead after 1999 commissioning, but was overtaken by KEKB in 2001. KEKB now has almost twice the accumulated luminosity, and is the "brightest" collider in the world. Between the two, a total of over $1000 \mathrm{fb}^{-1}\left(1 \mathrm{ab}^{-1}\right)$ data has been logged so far. Note that, at $\Upsilon(4 S)$ energy

$$
1 \mathrm{fb}^{-1} \cong 1.12 \mathrm{M} B \bar{B} \text { pairs, }
$$

together with about $1.3 \mathrm{M}(0.89 \mathrm{M}) c \bar{c}$ quark $\left(\tau^{+} \tau^{-}\right)$pairs, so a B factory is also a $\tau$ /charm factory. With $\Upsilon(4 S) \rightarrow B \bar{B} \cong 100 \%$ of the time, and the closeness of $B^{+}$and $B^{0}$ in mass, the amount of $B^{+} B^{-}$and $B^{0} \bar{B}^{0}$ are almost the same, and the fraction is measurable. With $\Upsilon(4 S)$ a $J=1$ resonance, the $B \bar{B}$ are flavor "locked", or "entangled" at production. This fact, that a $B$ decay at time $t$ (in CM frame) fixes the $\bar{B}$ flavor of the other $B$ at same time, is utilized in the mixing-decay interference study. The quantum entanglement effect has been recently studied and confirmed by Belle [13].

The Belle (see Fig. 1) and BaBar detectors have similar design concepts. From inside out: vertex detector, drift chamber, PID, ECAL, solenoid (1.5 Tesla), and flux return instrumented to detect muons and $K_{L}$. The main difference is in PID: Belle uses Aerogel Cherenkov Counters (plus TOF), a threshold device, while BaBar uses a quartz bar to extract Cherenkov light by total internal reflection, projecting into a water tank at the backside. Other differences are the 3 (later 4 ) layers vs 5 layers of $\mathrm{Si}$ for the vertex detector, and a closer (and no backward endcap) ECAL for Babar. 


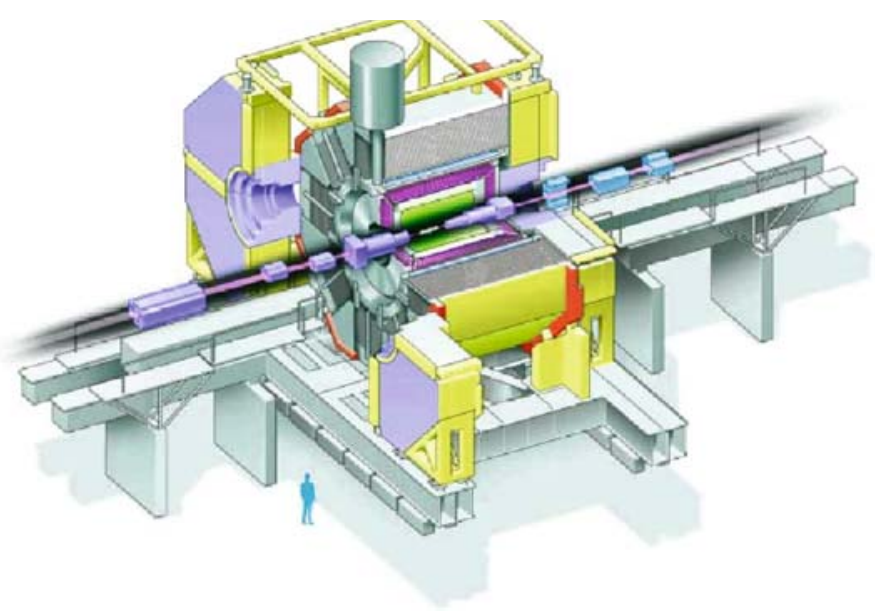

Fig. 1. The Belle detector.

Let us briefly summarize detection requirements for each subdetector. The SVD should have sufficient resolution for $\Delta z$ along the boost direction, which translates to $\Delta t$ of the two $B$ mesons. It must survive (longevity) and operate (tolerance) in a high radiation environment. The Belle SVD2 upgrade in 2003 improved the $1 \mathrm{MRad}$ radiation tolerance of SVD1 to better than $20 \mathrm{MRad}$, changed from 3 to 4 layers, and with improved angular coverage. Together with a smaller $1.5 \mathrm{~cm}$ (vs $2 \mathrm{~cm}$ ) beampipe radius, the IP (impact parameter) resolution was improved by over $60 \%$. Currently the SVD2 data fraction is already much larger than the original $152 \mathrm{M} B \bar{B}$ accumulated with SVD1.

The hadron PID, i.e. charged $\pi / K / p$ separation, is as important for CPV studies as vertexing. The lower momentum range is used for kaon tagging, while for two-body decay modes one needs $\pi^{ \pm} / K^{ \pm}$separation up to $4.4 \mathrm{GeV}$ (for Belle) in forward direction. A complication is operating phototubes in a strong B field. The Belle PID system consists of $d E / d x$ from CDC, TOF, and the ACC. The ACC is based on hydrophobic silica aerogel of ultra low density, with $n=1.01-1.028$ in barrel, to 1.03 in forward endcap. The DIRC detector of BaBar provides more information with lower pion fake rate.

The ECAL (called ECL at Belle) measures shower energy of $e, \gamma$ and $\pi^{0}$, gives best possible energy and position resolution, and optimal angular and energy coverage. Matching with tracker gives electron ID. As shown by CLEO II, the expense on crystals (thallium doped CsI) cannot be spared, but the cost, and the schedule for production, are limiting factors.

The low energy handicaps hadron calorimetry, but the iron flux return is instrumented (by layers of RPCs, or resistive plate chambers) to detect muons $(>800 \mathrm{MeV})$ by pairing with tracker. It also provides partial detection of $K_{L}$ mesons that largely penetrate all inner detectors, by pairing with ECL response. At Belle, this subdetector is called the KLM $\left(K_{L} /\right.$ Muon). 


\section{The Quest for CP Violation}

\subsection{CKM Unitarity Triangle}

With $\lambda, A$ and $\rho, \eta$ defined in Eq. (2), it is useful to give the Wolfenstein parametrization of the $3 \times 3$ quark mixing matrix,

$$
V=\left(\begin{array}{ccc}
1-\frac{1}{2} \lambda^{2} & \lambda & A \lambda^{3}(\rho-i \eta) \\
-\lambda & 1-\frac{1}{2} \lambda^{2} & A \lambda^{2} \\
A \lambda^{3}(1-\rho-i \eta) & -A \lambda^{2} & 1
\end{array}\right)+\mathcal{O}\left(\lambda^{4}\right)
$$

which puts the complex dynamics in the small, farthest off-diagonal element of $V_{u b}$ and $V_{t d}$, and is worth memorizing. The unitarity of $V$, i.e. $V^{\dagger} V=I$, is best visualized geometrically as a triangle, e.g. the $\left(V V^{\dagger}\right)_{d b}$ element is

$$
V_{u d} V_{u b}^{*}+V_{c d} V_{c b}^{*}+V_{t d} V_{t b}^{*}=0
$$

which is illustrated in Fig. 2. To over-constrain its angles and sides is the central theme at the $\mathrm{B}$ factories. The phase angles $\phi_{1}, \phi_{2}, \phi_{3}$, used by Belle are alternatively called $\beta, \alpha$ and $\gamma$ by BaBar.

\section{Tree Level B Decays}

The inclusive (quark level) diagram is given in Fig. 3(a), where we have suppressed the virtual $W$. The leading decays are $b \rightarrow c \bar{u} d, c \bar{c} s$, and the semileptonic $b \rightarrow c e \bar{\nu}, c \mu \bar{\nu}, c \tau \bar{\nu}$, proportional to $V_{c b}$; then the $\lambda$ suppressed $b \rightarrow c \bar{u} s$,

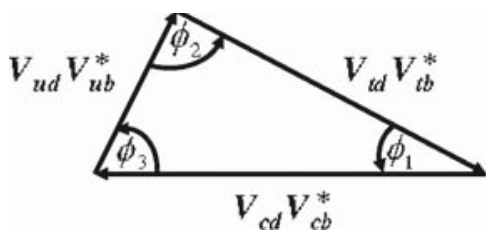

Fig. 2. UT triangle for Eq. (5).

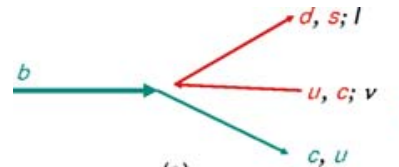

(a)

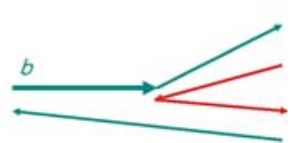

(b)

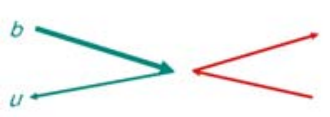

(c)

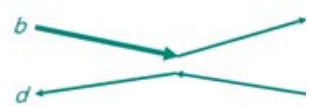

(d)

Fig. 3. Tree diagrams for $B$ decay. At exclusive level: (a) color allowed (external $W$ ), (b) color suppressed (internal $W$ ), (c) annihilation, (d) $W$ exchange. 
$c \bar{c} d$; the $V_{u b}$ suppressed $b \rightarrow u \bar{u} d, u \bar{c} s$, and semileptonic $b \rightarrow u e \bar{\nu}, u \mu \bar{\nu}, u \tau \bar{\nu}$. Finally, we have the $\lambda V_{u b}$ suppressed $b \rightarrow u \bar{u} s, u \bar{c} d$. The $V_{u b}$ related hadronic modes can probe the CPV phase via tree decays.

At the exclusive level, e.g. two body final states, we illustrate further in Fig. 3 how final state mesons may form. The color suppressed mode has color mismatch between, say the quark from $b$ decay and the spectator antiquark.

\subsection{CPV Primer: Direct CPV}

$\mathrm{CPV}$ is defined as a difference in probability between a particle process from the antiparticle process, e.g. between $B \rightarrow f$ and $\bar{B} \rightarrow \bar{f}$. It requires the presence of two interfering amplitudes, but besides the usual $i$ from quantum time evolution, it needs complex dynamics. That is, the interference involves the presence of two kinds of phases. Let us illustrate how CPV occurs.

Consider the particle amplitude $A=A_{1}+A_{2}$, where $A_{j}$ have a $C P$ invariant phase $\delta_{j}$ ( $i$ from QM) and a CPV phase $\phi_{j}$ ( $i$ from CPV dynamics). Absorbing an overall phase by defining $A_{1}=a_{1}$ to be real, one has

$$
\begin{aligned}
& A=A_{1}+A_{2}=a_{1}+a_{2} e^{i \delta} e^{+i \phi}, \\
& \bar{A}=\bar{A}_{1}+\bar{A}_{2}=a_{1}+a_{2} e^{i \delta} e^{-i \phi} .
\end{aligned}
$$

The $\delta$ and $\phi$ are called the "strong" and weak phase, respectively. The QM or strong phase $\delta$ does not distinguish between particle or antiparticle, hence sign is unchanged. However, the dynamical or weak phase $\phi$ changes sign for the antiparticle process $\bar{A}$. This enrichment of quantum interference leads to an asymmetry between particle and antiparticle probabilities,

$$
\mathcal{A}_{\mathrm{CP}} \equiv \frac{\Gamma_{\bar{B}^{0} \rightarrow \bar{f}}-\Gamma_{B^{0} \rightarrow f}}{\Gamma_{\bar{B}^{0} \rightarrow \bar{f}}+\Gamma_{B^{0} \rightarrow f}}=\frac{2 a_{1} a_{2} \sin \delta \sin \phi}{a_{1}^{2}+a_{2}^{2}+2 a_{1} a_{2} \cos \delta \cos \phi},
$$

defined with respect to quarks. As $\mathcal{A}_{\mathrm{CP}}$ vanishes with either $\delta$ or $\phi \rightarrow 0, \mathrm{CPV}$ requires the presence of both $C P$ conserving and $\mathrm{CPV}$ phases.

Eq. (6) is illustrated in Fig. 4, which shows geometrically how Eq. (7) materializes. If $\delta=0$, then $A_{1}+A_{2}$ and $\bar{A}_{1}+\bar{A}_{2}$ are at angle $\phi$ above or below the real axis, hence of equal length. If $\phi=0$, then $A_{1}+A_{2}$ and $\bar{A}_{1}+\bar{A}_{2}$

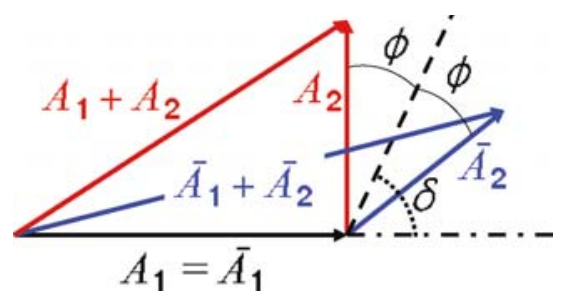

Fig. 4. Mechanism for CPV, Eqs. (6) and (7). 
(a)

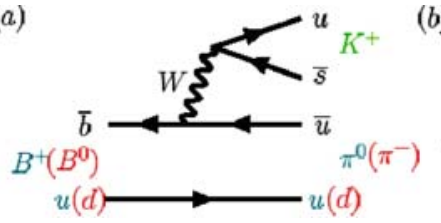

(b)

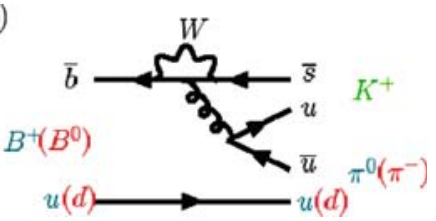

Fig. 5. Tree and Penguin $B \rightarrow K \pi, \pi \pi$ graphs.

are the same vector. Only when $\delta \neq 0$ and $\phi \neq 0$ does $\left|A_{1}+A_{2}\right| \neq\left|\bar{A}_{1}+\bar{A}_{2}\right|$ occur, which gives the asymmetry of Eq. (7).

In the KM model with 3 generations, the CPV phase is put in the 13 and 31 elements in the standard phase convention [6]. Thus, it is said that one needs the presence of all 3 generations to make CPV to occur.

\section{Direct CPV in $B^{0} \rightarrow K^{+} \pi^{-}$}

Direct CPV (DCPV), which has recently been established in $B^{0} \rightarrow K^{+} \pi^{-}$ (and $\pi^{+} \pi^{-}$as well) mode, gives the most intuitive illustration. In Fig. 5 we show the tree $(T)$ and penguin $(P)$ diagrams for $B \rightarrow K \pi, \pi \pi$ decays. The tree $b \rightarrow u \bar{s} u$ diagram carries a weak phase via $\arg V_{u s} V_{u b}^{*}$, while $P$ is dominated by $V_{c s} V_{c b}^{*} \cong-V_{t s} V_{t b}^{*}$, which is almost real. If $T$ and $P$ have a relative strong $\delta$ phase, their interference would lead to direct (i.e., in decay amplitude itself) CPV. Indeed this was observed in 2004 [14].

The whimsical name "penguin" is attributed to a bet by John Ellis 30 years ago. If one complains that Fig. 5(b) bears no resemblance to a "penguin", then neither does a Feynman diagram bear any resemblance to Feynman (although Feynman did pen it). We have already mentioned the discovery of the $B \rightarrow$ $K^{*} \gamma, X_{s} \gamma$ penguins by CLEO II in 1993. The exclusive strong penguin modes $B \rightarrow K \pi$ [15], $\eta^{\prime} K$ [16] were also discovered by CLEO II around 1998, and the strategy was clear that one searches for DCPV in rare decays. It took only 6 more years for DCPV in $B^{0} \rightarrow K^{+} \pi^{-}$to be measured between Belle and BaBar [14]. The electroweak penguin process $B \rightarrow K^{(*)} \ell^{+} \ell^{-}, X_{s} \ell^{+} \ell^{-}$, dominated by the $Z$ penguin [17], were discovered [18] by Belle in 2002 .

\section{Rare B Reconstruction}

DCPV can be observed only in rare processes. A standard procedure for rare $B$ reconstruction has been developed since the CLEO era for two body decays such as $B \rightarrow K \pi, \pi \pi$. One utilizes the special two body $\Upsilon(4 S) \rightarrow B \bar{B}$ decay kinematics: the $B$ energy is just $E_{\mathrm{CM}} / 2$, which is precisely known from the accelerator. Thus, the two standard variables are the energy difference $\Delta E$ and the beam constrained mass $M_{\mathrm{bc}}$,

$$
\Delta E=\sum E_{i}-E_{\mathrm{CM}} / 2, \quad M_{\mathrm{bc}}=\sqrt{\left(E_{\mathrm{CM}} / 2\right)^{2}-\sum\left(\boldsymbol{p}_{i}\right)^{2}},
$$


where $E_{i}$ and $\boldsymbol{p}_{i}$ are measured for particle $i$. A correctly reconstructed $B$ meson event would peak in $\Delta E$ and $M_{\mathrm{bc}}$, as can be visualized by $1 \mathrm{D}$ projection plots, while background events would not. Note that the $K$ and $\pi$ in $B \rightarrow K \pi$, $\pi \pi$ are rather highly boosted, hence PID (ACC at Belle and DIRC at BaBar) performance is rather critical to separate the $K \pi, \pi \pi$ events.

With these standard techniques, it was a matter of time and providence for one to eventually catch the first DCPV measurement, which happened to be the $B^{0} \rightarrow K^{+} \pi^{-}$mode. The summer 2006 combined result gives [19]

$$
\mathcal{A}_{K^{+} \pi^{-}}=-0.093 \pm 0.015,(\text { Summer 2006) }
$$

which is rather sizable, indicating a sizable $C P$ invariant phase $\delta$.

After CPV was discovered in $K^{0}$ mixing, it took 35 years for DCPV to be measured in kaon decay. For measurement of DCPV in $B$ decay, it took only 3 year after the measurement of $\mathrm{CPV}$ in $B^{0}$ mixing in 2001.

\subsection{Raison d'Être: $\sin 2 \phi_{1} / \beta$ from $B^{0} \rightarrow J / \psi K_{S}$}

The billion dollar question for the B factories is to measure CPV phase $\phi_{1} / \beta$ via $B^{0} \rightarrow J / \psi K_{S}$, which was fulfilled just 2 years after turning on!

We now need to explain the more intricate - and beautiful - mixingdecay interference [20]. We will identify what are $\delta$ and $\phi$ in Eqs. (6) and (7), the understanding of which should bring joy to the student.

\section{Mixing-decay Interference}

The final state $J / \psi K_{S}$ is a $C P$ eigenstate. For an initial $\bar{B}^{0}$ meson, there are two paths for it to decay to a $C P$ eigenstate $f_{\mathrm{CP}}$ : direct $\bar{B}^{0} \rightarrow J / \psi K_{S}$ decay (amplitude $A$ ), or via two steps, first $\bar{B}^{0} \rightarrow B^{0}$ through mixing, then $\bar{B}^{0} \rightarrow J / \psi K_{S}$ decay. In other words, denoting, the two paths $\bar{B}^{0} \stackrel{A}{\longrightarrow} J / \psi K_{S}$ and $\bar{B}^{0} \stackrel{M_{12}}{\longrightarrow} B^{0} \stackrel{\bar{A}}{\longrightarrow} J / \psi K_{S}$, where $M_{12}$ is the $\bar{B}^{0} \rightarrow B^{0}$ mixing amplitude, constitute the two interfering amplitudes $A_{1}$ and $A_{2}$ in Eq. (6).

From this we see that the $C P$ invariant phase is from $\bar{B}^{0} \leftrightarrow B^{0}$ oscillations, $e^{i \Delta m t}$, which is indeed quantum mechanical. The CPV weak phase comes from the dominant top contribution in the $b \bar{d} \rightarrow \bar{b} d$ box diagram, $M_{12} \propto V_{t d}^{* 2}$. The beauty of $\bar{B}^{0} \rightarrow J / \psi K_{S}$ decay is that, the dominant tree level $b \rightarrow c \bar{c} s$ amplitude is proportional to $V_{c s}^{*} V_{c b}$ and practically real. This is by convention of Eq. (4) and hard to be affected by New Physics. Even for the penguin analogue of Fig. 5(b), the CKM factor is $V_{t s}^{*} V_{t b} \cong-V_{c s}^{*} V_{c b}$, which is also practically real in SM. Therefore, using Belle notation, the weak phase $\phi$ is effectively $\arg V_{t d}^{* 2}=\sin 2 \phi_{1}$ to very good accuracy within SM. This is the reason why the measurement of time-dependent (mixing or oscillation dependent) CPV, or TCPV, became the raison d'être for the asymmetric B factory, and why good vertexing was placed at a premium. 
Without further ado, we give the refinement of Eq. (7) for TCPV study in $B^{0}$ decays to $C P$ eigenstate $f[6]$ :

$$
A(\Delta t) \equiv \frac{\Gamma_{\bar{B}^{0}(\Delta t) \rightarrow f}-\Gamma_{B^{0}(\Delta t) \rightarrow f}}{\Gamma_{\bar{B}^{0}(\Delta t) \rightarrow f}+\Gamma_{B^{0}(\Delta t) \rightarrow f}}=-\xi_{f}\left(\mathcal{S}_{f} \sin \Delta m \Delta t+\mathcal{A}_{f} \cos \Delta m \Delta t\right),
$$

where $\xi_{f}$ is the $C P$ eigenvalue of $f, \Delta m=\Delta m_{B_{d}}, \bar{B}^{0}(\Delta t)$ is the state at time $\Delta t$ for $\bar{B}^{0}(0)=\bar{B}^{0}$, and $\Delta t=t_{\mathrm{CP}}-t_{\mathrm{tag}}$ (more detail later), the time difference between " $C P$ " side (e.g. $J / \psi K_{S}$ ) and flavor tagged side. The coefficient $\mathcal{S}_{f}$ associated with $\sin \Delta m \Delta t$ corresponds to $\sin \phi$ in Eq. $(7)$, and $\mathcal{A}_{f}\left(-\mathcal{C}_{f}\right.$ for BaBar) associated with cos $\Delta m \Delta t$ oscillation is related to DCPV, which could occur even at $\Delta t=0$ (i.e. without oscillations). They are given by

$$
\mathcal{S}_{f}=\frac{2 \operatorname{Im} \lambda_{f}}{\left|\lambda_{f}\right|^{2}+1}, \quad \mathcal{A}_{f}=\frac{\left|\lambda_{f}\right|^{2}-1}{\left|\lambda_{f}\right|^{2}+1}, \quad \text { where } \lambda_{f} \equiv \frac{q}{p} \frac{\left\langle f|S| \bar{B}^{0}\right\rangle}{\left\langle f|S| B^{0}\right\rangle} .
$$

Clearly one has $\mathcal{A}_{f}=\mathcal{A}_{\mathrm{CP}}$. Since $\left\langle f|S| \bar{B}^{0}\right\rangle$ is real for $f=J / \psi K_{S}$, one gets $\lambda_{f} \cong e^{-2 i \phi_{1}}$, so $T C P V$ in $\bar{B}^{0} \rightarrow J / \psi K_{S}$ is free of hadronic uncertainties.

\section{Measurement of TCPV in $b \rightarrow c \bar{c} s$}

The $\Upsilon(4 S)$ is only about $20 \mathrm{MeV}$ above the $B \bar{B}$ threshold, so the transverse motions of the $B$ 's are insignificant. The vertex detector measures both decay vertices along the $z$ axis, defined as the high momentum beam direction, and $\Delta t \cong \Delta z / \beta \gamma c$. The "CP side" is reconstructed in, say, $J / \psi K_{S}$, the cleanest mode both experimentally and theoretically. The flavor has to be inferred from the "tag side", where one uses coherence to assign opposite flavor of tag side at $\Delta t=t_{\mathrm{CP}}-t_{\mathrm{tag}}=0$. One then uses the time evolution of Eq. (10) to fit for $\mathcal{S}_{f}$ and $\mathcal{A}_{f}$. To summarize, one needs to

1) Reconstruct one $B$ in $C P$ eigenstate,

2) Tag the other $B$ flavor,

3) Measure both decay vertices.

The effort is to reconstruct as many $b \rightarrow(c \bar{c})_{\text {charmonium }} s$ final states with definite $C P$, including angular resolved $B \rightarrow J / \psi K^{*}$ to disentangle $C P$ components. Correcting for $\xi_{f}$, one enlarges statistics to measure the so-called $\mathcal{S}_{c \bar{c} s}$, assuming that hadronic and other corrections are small.

$B \rightarrow J / \psi K_{S} \rightarrow \ell^{+} \ell^{-} \pi^{+} \pi^{-}$final state is the easiest and cleanest to reconstruct because of the very narrow $J / \psi$ width. Next, $B \rightarrow J / \psi K_{L}$ is reconstructed by associating activities in the KLM detector (IFR at BaBar) with no tracks (and potentially some activity in ECL), but the $S / B$ gets worse. Correcting for the $\xi_{f}$ factor, the combined result is called $\mathcal{S}_{J / \psi K^{0}}$. The collected events with a dataset of $535 \mathrm{M} B \bar{B}$ pairs by Belle is shown in Fig. 6, where $B \rightarrow J / \psi K_{S}$ is evidently much cleaner. One also reconstructs in $\psi^{\prime} K_{S}$, $\eta_{c} K_{S}, \chi_{c 1} K_{S}$ as well as $J / \psi K^{* 0}$ (with flavor nonspecific $K^{* 0}$ decays).

For flavor tagging, semileptonic decay provides the cleanest tag, but other tags, such as kaon (even $\Lambda$ ) flavor from the $b \rightarrow c \rightarrow s$ decay chain are 

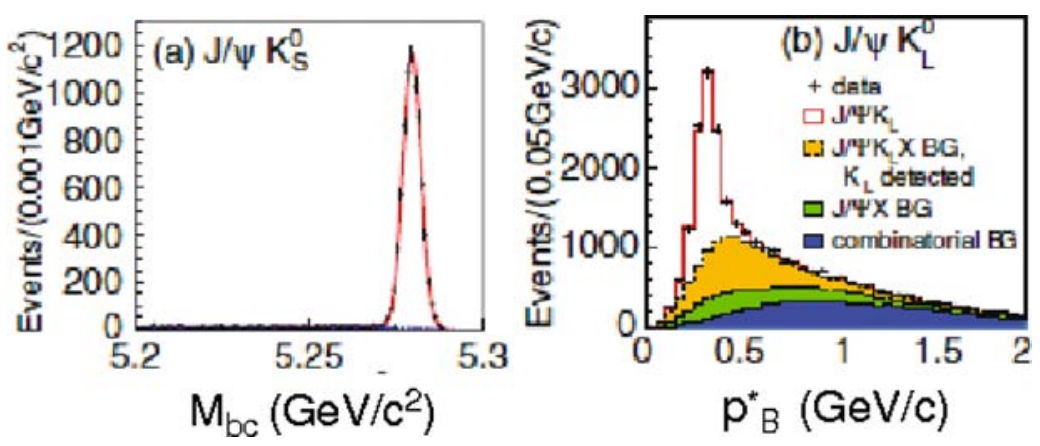

Fig. 6. (a) $M_{\mathrm{bc}}$ distribution of $B^{0} \rightarrow J / \psi K_{S}^{0}$ candidate events in $\Delta E$ signal region, and (b) $p_{B}^{*}(\mathrm{CM})$ distribution for $B^{0} \rightarrow J / \psi K_{L}^{0}$ candidate events.

used, which has higher efficiency than lepton tags. The mistag probability $w$ is defined as 1 minus the correctness of tag, which has to be measured, leading to a dilution factor $D=1-2 w$. At Belle one produces the $q=+/-$ assignment $\left(B^{0} / \bar{B}^{0}\right.$ on tag side), with a purity of tag $r \in(0,1)$.

Blind Analysis is worth special emphasis for B factories (and for all precision measurements, especially those with sign ambiguities). That is, the analysis is done "blind" to avoid possible bias. The to-be measured quantity is shifted randomly by some amount (within range of a few expected errors). All systematic studies are completed before "unblinding" for the final measurement.

The measurement of $\sin 2 \phi_{1} / \beta$ ( $\equiv \mathcal{S}_{c \bar{c} s}$ by the assumption that corrections are small) is as fundamental as the fine structure constant $\alpha$. The world history of $\sin 2 \phi_{1} / \beta$ measurement for 1998-2005 is shown in Fig. 7. The raison d'être for the B factories was achieved in year 2001 .

To get a feel of data quality and current status, we plot in Fig. 8 the result for $B^{0} \rightarrow J / \psi K^{0}$ with $535 \mathrm{M} B \bar{B}$ pairs collected by Belle. Note the consistency between $B^{0} \rightarrow J / \psi K_{S}^{0}$ and $J / \psi K_{L}^{0}$. The measured value is $\sin 2 \phi_{1}=0.642 \pm 0.034 \pm 0.017$ for Belle, which is only for $B^{0} \rightarrow J / \psi K^{0}$. BaBar continued to combine all $b \rightarrow c \bar{c} s$ modes, and the 2006 result is $\sin 2 \beta=0.714 \pm 0.032 \pm 0.018$, combining with Belle to $0.678 \pm 0.026$. Combining further with all measurements, the current world average is [19]

$$
\sin 2 \phi_{1} / \beta=0.678 \pm 0.025, \quad(\text { Winter 2007) }
$$

which now has a precision of $4 \%$. This gives

$$
\phi_{1} / \beta=(21.3 \pm 1.0)^{\circ}, \quad(\text { Winter 2007) }
$$

up to sign ambiguities ( $68.7^{\circ}$ is in principle allowed). Hence, $\sin 2 \phi_{1} / \beta$ has now been precisely measured, with no sign of deviation (?) from SM, but note that there is some variance between the Belle and BaBar results. 


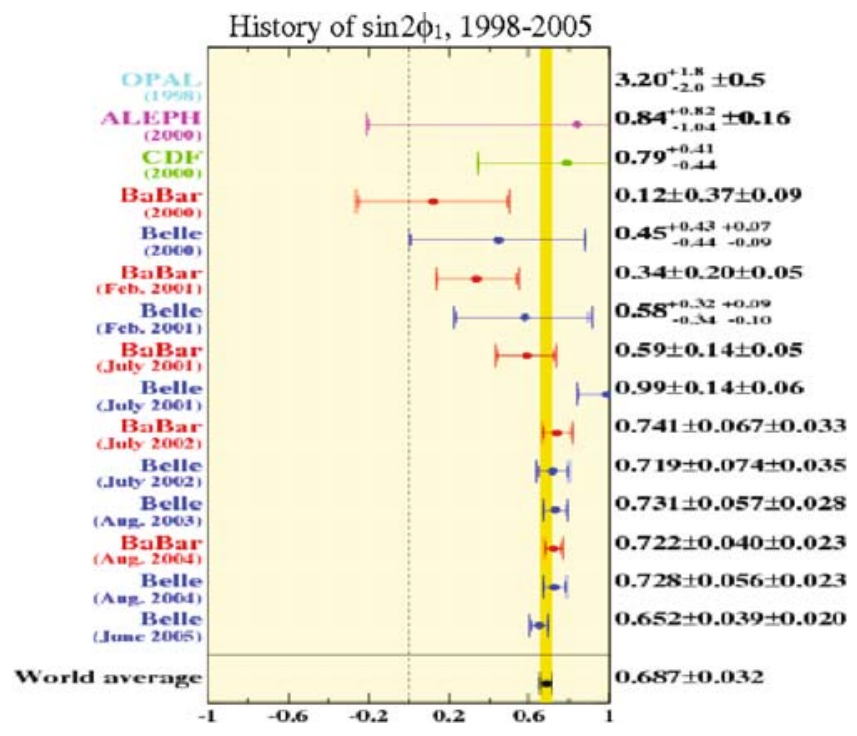

Fig. 7. World history of $\sin 2 \phi_{1} / \beta$ measurement.
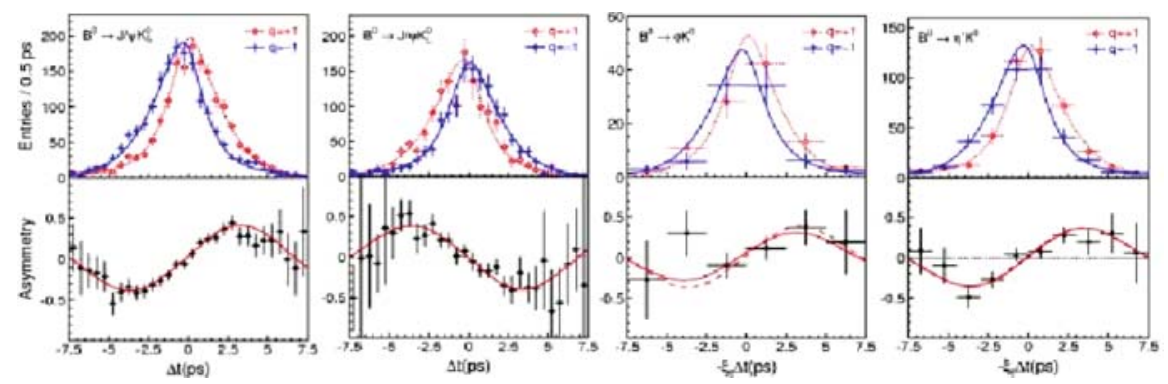

Fig. 8. Background-subtracted $\Delta t$ distributions and asymmetries for (a), (b) $B^{0} \rightarrow$ $J / \psi K^{0}$ events of Fig. 6 , and (c) $\phi K^{0}$, (d) $\eta^{\prime} K^{0}$, with good tags $(r>0.5)$.

The question mark above refers to the tension between Eq. (12) and e.g. the indirect "measurement" of $\sin 2 \phi_{1} / \beta=0.752_{-0.035}^{+0.057}$ (Spring 2006) by fitting [21] to all data without the direct measurement input, which heightens if one takes the Belle result. Taken seriously, this could indicate New Physics.

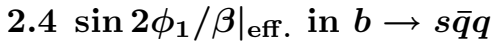

Possible hints for New Physics emerged in the $\mathcal{S}_{f}$ parameter measurement in $b \rightarrow s \bar{q} q$ modes, which are dominated by the strong penguin $P$.

The excitement began with the summer 2003 result from Belle, based on $140 \mathrm{fb}^{-1}$ data collected with the SVD1 vertex detector. With $68 \pm 11$ reconstructed $B \rightarrow \phi K_{S}$ events, Belle measured $\mathcal{S}_{\phi K_{S}}=-0.96 \pm 0.50_{-0.11}^{+0.09}$, 
which is rather different from $\mathcal{S}_{c \bar{c} s}$ value of Eq. (12), or the world average of 2003. However, dominated by a diagram like Fig. 5(b) with $g^{*} \rightarrow u \bar{u}$ replaced by an $s \bar{s}$ pair, the penguin amplitude $\propto V_{t s}^{*} V_{t b}$ in SM is also practically real, hence $\lambda_{\phi K_{S}} \cong q / p$ just as for $B \rightarrow J / \psi K_{S}$, hence

$$
\mathcal{S}_{\phi K_{S}} \cong \sin 2 \phi_{1} / \beta, \quad(\mathrm{SM})
$$

is predicted. The Belle result showed a large deviation, and even the sign was opposite. Though not yet statistically significant enough (and with BaBar at variance), it aroused great interests, and many speculations about whether NP FCNC and CPV loop effects could break the equality of Eq. (14).

The large deviation indicated by Belle 2003 data turned out to be a mirage. By 2004, with doubling of the data, the measured value for $\mathcal{S}_{\phi K_{S}}$ vanished. It so happened that Belle installed the upgraded SVD2 vertex detector in summer 2003, and the almost equal amount of SVD2 data had opposite sign with the earlier SVD1 data. This does not mean the earlier data was wrong, since the number for that dataset did not change upon reanalysis. Statistical fluctuations do happen, and the only antidote is to have more data!

By 2006, the Belle analysis included $K_{S} \rightarrow \pi^{0} \pi^{0}$ as well as $K_{L}$, including $\phi \rightarrow K_{S} K_{L}$ reconstruction. Out of $535 \mathrm{M} B \bar{B}$ pairs, about $310 B \rightarrow \phi K_{S}$ and $110 \phi K_{L}$ events were reconstructed. The $S / B$ is of course much poorer than Fig. 6. We give the $\Delta t$ distribution and asymmetry in Fig. 8(c), which should be compared with Fig. 8(a). The measured result is

$$
\mathcal{S}_{\phi K^{0}}=+0.50 \pm 0.21 \pm 0.06, \quad(\text { Belle } 535 \mathrm{M})
$$

which is $1 \sigma$ below Eq. (12), but still consistent with Eq. (14). The BaBar 2005 result gives $+0.50 \pm 0.25_{-0.04}^{+0.07}$, but a different method was adopted in 2006. Fitting to low mass $K^{+} K^{-}$region for $\phi K^{0}$ and $f_{0}(980) K^{0}$ of three body $K^{+} K^{-} K^{0}$, gives $\mathcal{S}_{\phi K^{0}}=+0.12 \pm 0.31 \pm 0.10$, which is lower than Eq. (15).

Fig. 8(d) shows the $B^{0} \rightarrow \eta^{\prime} K^{0}$ result for Belle, giving

$$
\mathcal{S}_{\eta^{\prime} K^{0}}=+0.64 \pm 0.10 \pm 0.04, \quad(\text { Belle } 535 \mathrm{M})
$$

which, at $5.6 \sigma$ this is the first observation of TCPV in a single charmless $b \rightarrow s$ mode. It is fully consistent with SM, i.e. the relation similar to Eq. (14). Unlike the large early fluctuation in $\phi K^{0}$ mode, here Belle has measured consistent results throughout. With $347 \mathrm{M} B \bar{B}$ pairs, BaBar measures $\mathcal{S}_{\eta^{\prime} K^{0}}=$ $+0.55 \pm 0.11 \pm 0.02$, a $4.9 \sigma$ effect, but it has migrated from a value consistent with zero in early phase, through the 2005 result of $+0.36 \pm 0.13 \pm 0.03$.

\section{The $\Delta \mathcal{S}$ Problem}

Many other $b \rightarrow s \bar{q} q$ modes are studied. No significant results for $\mathcal{A}_{f}\left(=-\mathcal{C}_{f}\right)$ have been established so far. The emerging picture is a consistent systematic shift of $\mathcal{S}_{f}$ in these modes towards the lower side of $\mathcal{S}_{c \bar{c} s}$. In Fig. 9 we plot the 


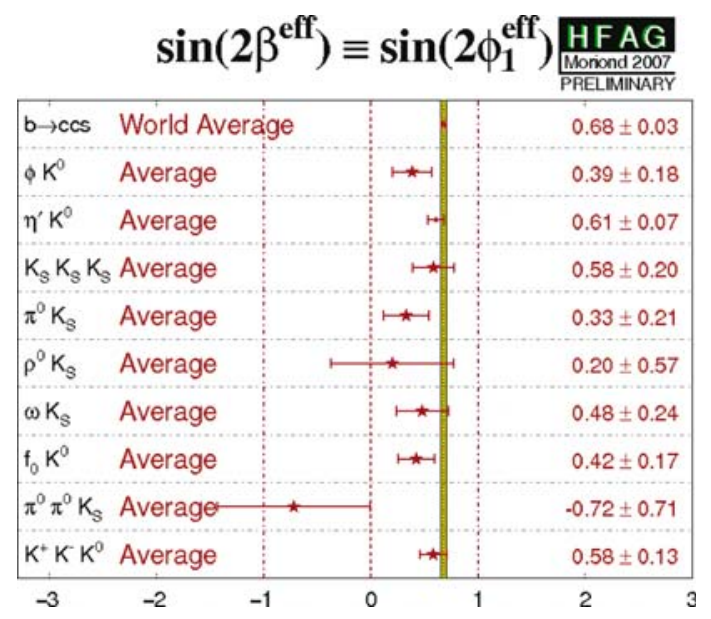

Fig. 9. HFAG Winter 2007 average result for $\mathcal{S}_{s \bar{q} q}$.

HFAG average results for $\mathcal{S}_{f}$ in various charmless $b \rightarrow s \bar{q} q$ modes, compared with $b \rightarrow c \bar{c} s$ (first row). All 9 charmless modes are lower than $\mathcal{S}_{c \bar{c} s}$, and echoes the original Belle splash of 2003. The naive average gives

$$
\mathcal{S}_{s \bar{q} q}=+0.53 \pm 0.05, \quad \text { (Winter 2007) }
$$

which can be compared with Eq. (12). In contrast, considering corrections to Eq. (14), theory expects a positive shift for SM. Given that the over $-2 \sigma$ difference between $\mathcal{S}_{s \bar{q} q}$ and $\mathcal{S}_{c \bar{c} s}$ has persisted for several years, and with Belle and BaBar generally in agreement, either there is some common experimental systematics, or it could be taken as a possible hint for New Physics. This has been one of the most important outcomes from B factories.

Again, more data would be needed, requiring something beyond $\mathrm{LHCb}$, such as a Super B factory.

\section{$2.5 \sin 2 \phi_{2} / \alpha$ in $b \rightarrow u \bar{u} d$}

A clean measurement for $\phi_{2} / \alpha$ was originally projected, when the $b \rightarrow d \bar{u} u$ penguins, the analogues of $b \rightarrow s \bar{u} u$ were ignored. If so, then the formalism of Eqs. (10)-(11) would hold, just like for $b \rightarrow c \bar{c} s$ and $s \bar{q} q$, except that now one does have CPV phase in the decay amplitude, e.g. $\left\langle\pi^{+} \pi^{-}|S| \bar{B}^{0}\right\rangle /\left\langle\pi^{+} \pi^{-}|S| B^{0}\right\rangle$ $\cong V_{u b} / V_{u b}^{*}=e^{-2 i \phi_{3}}$. Combining with $q / p$, one gets $\lambda_{\pi^{+} \pi^{-}}=e^{+2 i \phi_{2}}$, where $\phi_{1}+\phi_{2}+\phi_{3}=\pi$ has been used.

\section{$\pi \pi$ Program}

Alas, the penguin cannot be ignored. Furthermore, there may be a strong phase $\delta$ between the tree and penguin amplitudes. The diagrams are just as in Fig. 5 except replacing $\bar{s}$ by $\bar{d}$. Retracing the above steps, one gets 


$$
\lambda_{\pi^{+} \pi^{-}}=e^{+2 i \phi_{2}} \frac{T+P e^{+i \phi_{3}} e^{i \delta}}{T+P e^{-i \phi_{3}} e^{i \delta}},
$$

which collapses back to $e^{+2 i \phi_{2}}$ as $P / T \rightarrow 0$. But it seems that $P / T \sim 0.3$, as can be seen from comparing the penguin dominant $B \rightarrow K \pi$ rate with the tree dominant $B \rightarrow \pi \pi$ rate, taking care of CKM factors. Furthermore, the presence of both weak and $\delta$ phases leads to DCPV, i.e. the prospect for a sizable $\mathcal{A}_{f}$ term in the TCPV fit. Thus, the $\pi^{+} \pi^{-}$analysis would lead to only

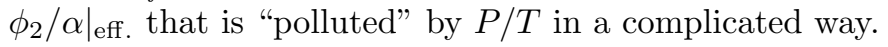

Many improved methods were proposed to alleviate the problem. Eventually one needs to do an isospin, or SU(2), analysis by combining all information, both decay rate and $C P$ asymmetries, from $\pi^{+} \pi^{-}, \pi^{+} \pi^{0}$ as well as $\pi^{0} \pi^{0}$ modes. This approach can remove the "penguin pollution" and disentangle $\phi_{2} / \alpha$ from $\phi_{2} /\left.\alpha\right|_{\text {eff. }}$. The discovery of sizable enhancement of the color-suppressed (see Fig. 3(b)) $\pi^{0} \pi^{0}$ mode gave hope to this program.

The measurement of $\phi_{2} / \alpha$, starting with the $\pi^{+} \pi^{-}$mode, has a tortuous history to match theory development, and Belle and BaBar have never shown good agreement. The current Belle result is

$$
\begin{aligned}
& \mathcal{S}_{\pi \pi}=-0.61 \pm 0.10 \pm 0.04, \quad(\text { Belle } 535 \mathrm{M}) \\
& \mathcal{A}_{\pi \pi}=+0.55 \pm 0.08 \pm 0.05
\end{aligned}
$$

based on $\sim 1460$ signal events, claiming $5.5 \sigma$ and $5.6 \sigma$ observations for DCPV and TCPV, respectively. BaBar gives

$$
\begin{aligned}
& \mathcal{S}_{\pi \pi}=-0.60 \pm 0.11 \pm 0.03,(\text { BaBar } 383 \mathrm{M}) \\
& \mathcal{A}_{\pi \pi}=+0.21 \pm 0.09 \pm 0.02
\end{aligned}
$$

based on $\sim 675 \pi^{+} \pi^{-}$events, which give $5.5 \sigma$ evidence for overall $\mathrm{CPV}$, but only a mild hint for $\operatorname{DCPV}\left(\mathcal{A}_{\pi \pi}\right)$. We plot the history of $\pi^{+} \pi^{-}$study in Fig. 10. It started with strong disagreement: Belle having a strong effect outside the physical boundary, while BaBar started consistent with zero. Now there is consensus that $\mathcal{S}_{\pi \pi} \sim-0.60$, but disagreement remains in $\mathcal{A}_{\pi \pi}$. It is said that, by $\mathrm{SU}(3)$ symmetry, the DCPV in $B^{0} \rightarrow \pi^{+} \pi^{-}$should be roughly 3 times as large as in $B^{0} \rightarrow K^{+} \pi^{-}$. If such is the case, then the mean of the Belle and BaBar results seem correct.

The $\pi \pi$ program for $\phi_{2} / \alpha$ measurement still lacks $\mathcal{S}_{\pi^{0} \pi^{0}}$ and $\mathcal{A}_{\pi^{0} \pi^{0}}$. No stringent constraint is obtained with $\pi \pi$ alone.

\section{$\rho \pi$ Program}

It had been claimed that an analysis in $B^{0} \rightarrow \pi^{+} \pi^{-} \pi^{0}$ Dalitz plot, by interference between $\rho^{ \pm} \rho^{\mp}$ and $\rho^{0} \pi^{0}$ modes, could give a clean measure of $\phi_{2} / \alpha$. The simplified early assumptions turned out also to be a false start.

In fact, the $\rho \pi$ program can be pursued, but the analysis is extremely complicated. We just mention that the latest study involves 26 fit parameters. 

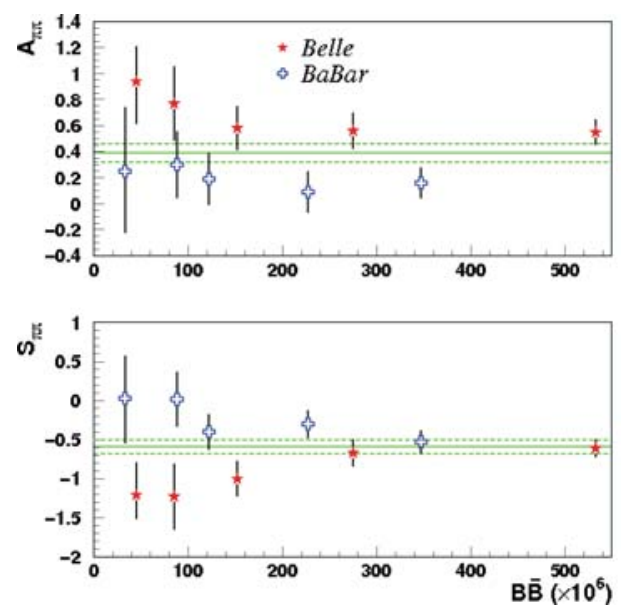

Fig. 10. History of TCPV study in $\pi^{+} \pi^{-}$mode.

The Belle result based on $449 \mathrm{M} B \bar{B}$ pairs with a Dalitz plot isospin analysis, give $67^{\circ}<\phi_{2}<95^{\circ}$, but other regions are allowed. The BaBar result, based on $375 \mathrm{M} B \bar{B}$ pairs, gives $\alpha=\left(87_{-13}^{+45}\right)^{\circ}$, with no constraint at $2 \sigma$.

\section{$\rho \rho$ Program}

With the difficulties in $\pi \pi$ and $\rho \pi$ programs, for some time the $\rho \rho$ program seemed the best $\phi_{2} / \alpha$ program before 2006: $\mathcal{B}\left(B^{0} \rightarrow \rho^{+} \rho^{-}\right)$is large, almost $100 \%$ longitudinally polarized hence a pure $C P$ eigenstate with no need for angular disentanglement. $\mathcal{B}\left(\rho^{0} \rho^{0}\right)$ seemed very small, hence no need for isospin analysis. Surprisingly, unlike $\rho \pi$ or modes involving kaons, there is little 3 - or 4-body contamination by resonances. With such blessings, the summer 2004 and 2005 results, dominated by the BaBar study, were quite consistent with CKM fit results made without information of $\alpha$ in the fit.

Alas, things made the turn for the worse in 2006: BaBar found $3.5 \sigma$ evidence for $\rho^{0} \rho^{0}$, and the situation became somewhat analogous to the $\pi \pi$ program, and the constraint on $\phi_{2} / \alpha$ weakened to $92 \pm 21$ degrees, and $\rho \rho$ is no longer the "best program". One now needs information on $\mathcal{A}_{\rho^{0}} \rho^{0}$.

The combined result from $\pi \pi / \rho \pi / \rho \rho$ does give a useful result on $\phi_{2} / \alpha$. With the Belle $\rho \pi$ result yet to be included, one has

$$
\phi_{2} / \alpha=\left(93_{-9}^{+11}\right)^{\circ}
$$

which can be compared with the global fit result of $\left(98_{-19}^{+5}\right)^{\circ}$ without information input from $\phi_{2} / \alpha$ itself.

With all its complexity and uncertainties, the $\phi_{2} / \alpha$ program is not a good place to discern New Physics effects. Discussion of measurement of the third phase $\phi_{3} / \gamma$ is deferred to Lecture 3 . 


\subsection{New Physics in DCPV?}

Regarding New Physics, we remark in passing that there may be a second hint for New Physics from the B factories, namely the aforementioned DCPV discussed in $\S 2.6$. Concurrent with the measurement of $\mathcal{A}_{K^{+} \pi^{-}}$, Eq. (9), it was found that the behavior for $B^{+} \rightarrow K^{+} \pi^{0}$ was different

$$
\mathcal{A}_{K^{+} \pi^{0}}=+0.047 \pm 0.026, \quad(\text { Summer 2006) }
$$

which is consistent with zero, even opposite in sign to $\mathcal{A}_{K^{+} \pi^{-}}$. The difference

$$
\Delta \mathcal{A}_{K \pi} \equiv \mathcal{A}_{K^{+} \pi^{0}}-\mathcal{A}_{K^{+} \pi^{-}}=0.14 \pm 0.03
$$

is more than $5 \sigma$ from zero. This certainly has something to do with enhancement of color-suppressed tree diagrams (Fig. 3(b)), as we have seen in $B^{0} \rightarrow \pi^{0} \pi^{0}$. But it could also arise partially from New Physics through the electroweak penguin $P_{\text {EW }}$ process that contributes to $B^{+} \rightarrow K^{+} \pi^{0}$. The electroweak penguin is dominated by $Z$, or $b s Z$ coupling. The virtual $Z^{0}$ can turn into a $\pi^{0}$ but not $\pi^{ \pm}$, so differentiates between $\mathcal{A}_{K^{+} \pi^{0}}$ and $\mathcal{A}_{K^{+} \pi^{-}}$. Since the effective $b s Z$ coupling within SM is real, to be able to generate Eq. (23) would require New Physics CPV!

\section{A Broadband Pursuit of FPCP}

Having covered the main part of CPV, in this section we cover many different topics to illustrate the richness of flavor physics explored at the B factories and beyond. Each subject can be touched upon only briefly.

\subsection{Sides of Unitarity Triangles: $V_{c b}$ and $V_{u b}$}

The pattern $\left|V_{u b}\right|^{2} \ll\left|V_{c b}\right|^{2} \ll\left|V_{u s}\right|^{2}$ lead to the Wolfenstein parametrization of Eq. (4). Measurement of $V_{c b}$ is equivalent to measurement of $A$, while $V_{u b}$ gives $\rho$ and $\eta$, the latter can only be probed via CPV effects.

The precision of $V_{c b}$ measurement was greatly improved by Heavy Quark Symmetry [22], which is rather analogous to heavy nucleus effect in atomic physics. The identical chemistry of isotopes corresponds to flavor symmetry, while the minuscule "hyperfine" splittings correspond to spin symmetry.

Imagine $B \rightarrow D^{*} \ell \nu$ decay. In the $m_{b}, m_{c} \rightarrow \infty$ limit, when a $b$ quark decays to $c \ell \nu$, if the $c$ quark remains stationary, while the $\ell$ and $\nu$ exit the meson practically without interacting, the "brown muck" or light degrees of freedom of the meson is unaffected. This zero-recoil limit corresponds to $w=1$, where $2 w m_{B} m_{D^{*}}=m_{B}^{2}-m_{D^{*}}^{2}-q^{2}$ with $q^{2}$ the lepton pair mass squared. In formula analogous to muon decay, one has a $B \rightarrow D^{*}$ "form 
factor" $F(w)$ that depends only on $w$ in the heavy quark limit, and computable by lattice QCD. At the inclusive level, Luke's theorem says $1 / m_{b}$ correction vanishes. These and other theoretical tools aid the precise determination of $V_{c b}$. But the actual discussion quickly gets technical. For all practical purposes (FAPP), $\left|V_{c b}\right| \simeq 0.04$, and $A \simeq 0.85$.

The measurement of $\left|V_{u b}\right|$ is much more difficult. The $b \rightarrow u \ell \nu$ rate is only at \% level of $b \rightarrow c \ell \nu$, which becomes the main background. Proposed inclusive methods have been to impose cuts on the $E_{\ell}, m_{X}$ or $q^{2}$ spectrum above the $m_{D}$ related threshold. Alternatively, one could measure exclusive $B \rightarrow(\pi, \rho, \omega) \ell \nu$ modes and face form factor uncertainties, with less aid from Heavy Quark Symmetry. At the moment, inclusive methods tend to give higher values than exclusive methods. FAPP for the nonspecialist, $\sqrt{\rho^{2}+\eta^{2}} \sim 0.5$.

\subsection{CPV Phase $\phi_{3} / \gamma: D K$ and $D \pi$ Methods}

Many methods have been proposed for the measurement of the third CPV phase angle $\phi_{3}$, or $\gamma$, of the unitarity triangle of Fig. 2 that corresponds to Eq. (5). But the most promising method in the long term, is the so-called " $D K$ " method, based on interference between two tree diagrams.

Quantum interference between two amplitudes is always the key to the study of CPV, as illustrated in $\$ 2.2$ and Fig. 4. Here one has two tree amplitudes, so insensitive to New Physics effect. Consider $B^{-} \rightarrow D^{(*) 0} K^{(*)-}$, $\bar{D}^{(*) 0} K^{(*)-}$ decays. The first arises from CKM-favored $b \rightarrow c \bar{u} s$ (Fig. 3(a)) process $\propto V_{c b} V_{u s}^{*}=A \lambda^{3}$, while the second arises from the CKM- and colorsuppressed $b \rightarrow u \bar{c} s$ (Fig. 3(b)) process $\propto V_{u b} V_{c s}^{*}=A \lambda^{3}(\rho-i \eta)$. The two amplitudes interfere when $D^{(*) 0}$ and $\bar{D}^{(*) 0}$ decay to common final state $f$. Depending on $f$, three methods have been proposed to exploit this interference: GLW ( $D^{0}$ decay to $C P$ eigenstate), ADS (doubly Cabibbo suppressed $D^{0}$ decay) and "Dalitz plot analysis". We shall present only the latter [23], because the second amplitude is very suppressed hence requires good statistics. Furthermore, it is the most experimentally oriented.

Let us illustrate with $D^{0} \rightarrow K_{S} \pi^{+} \pi^{-}$, which has a large branching fraction. $B^{\mp} \rightarrow\left[K_{S} \pi^{+} \pi^{-}\right]_{D} K^{\mp}$ decay can be described by the amplitude,

$$
M_{\mp} \propto f\left(m_{\mp}^{2}, m_{ \pm}^{2}\right)+r e^{i \delta} e^{\mp i \phi_{3}} f\left(m_{ \pm}^{2}, m_{\mp}^{2}\right)
$$

where $A\left(B^{-} \rightarrow \bar{D}^{0} K^{-}\right) / A\left(B^{-} \rightarrow D^{0} K^{-}\right) \equiv r e^{i \delta} e^{-i \phi_{3}}$, and $f\left(m_{-}^{2}, m_{+}^{2}\right)$ describes the Dalitz plot amplitude for $D^{0} \rightarrow K_{S} \pi^{+} \pi^{-}$, with $m_{ \pm}^{2} \equiv m^{2}\left(K_{S} \pi^{ \pm}\right)$. The latter can be determined from flavor-tagged $D^{*+} \rightarrow D^{ \pm} \pi^{+}$decays, but requires some modelling. With very large data sample, a bin-by-bin analysis [23] can remove model dependence. We stress again that the method is powerful because $r$ is very small, and the method includes GLW and ADS regions. Experimental studies pair up $D, D^{*}$ and $K, K^{*}$ modes.

An analogous approach is the $D \pi$ method for $2 \phi_{1}+\phi_{3}$. This process involves the allowed $b \rightarrow c \bar{u} d$ decay, interfering with a $\lambda V_{u b}$ suppressed $\bar{b} \rightarrow \bar{u} c \bar{d}$ 
decay brought about by $B^{0}-\bar{B}^{0}$ mixing, hence the extra $2 \phi_{1}$ factor. Here $r$ is extremely suppressed, hence the method is less fruitful at present.

The combined result on $\phi_{3}$ gives

$$
\phi_{3} / \gamma=\left(71_{-30}^{+22}\right)^{\circ}
$$

which is the least certain among the three CPV phases. Adding up Eqs. (13), (21) and (25), one has

$$
\phi_{1}+\phi_{2}+\phi_{3} \sim 185^{\circ}
$$

which is consistent with closing, but still with large errors. One would need LHCb and likely a Super B factory to reach convincing precision that Eq. (5) indeed closes as a triangle.

\subsection{Measurement of Large $B_{s}$ Mixing (vs $b \rightarrow d$ Penguins)}

Ever since the observation of large $B^{0}$ mixing, it was clear that $B_{s}-\bar{B}_{s}$ oscillations would be very rapid hence hard to measure. There is at least the enhancement factor of $\left|V_{t s} / V_{t d}\right|^{2} \sim \lambda^{-2} \sim 21$. At the end of the LEP era (the rapid oscillations make measurement at $\mathrm{B}$ factories infeasible), the lower bound for $\Delta m_{B_{s}}$ was roughly at $14 \mathrm{ps}^{-1}$, or about 18 times $\Delta m_{B_{d}}$.

The Tevatron Run II should have the capability to probe the SM range of $15-30 \mathrm{ps}^{-1}$. It was finally delivered in 2006 . With the first hint from $\mathrm{D} \emptyset$, the CDF experiment reported evidence, then observation [24], of $B_{s}$ mixing,

$$
\Delta m_{B_{s}}=17.77 \pm 0.10 \pm 0.07 \mathrm{ps}^{-1},
$$

which is already a precision measurement. The result, using the amplitude scan method (a filter), is shown in Fig. 11.

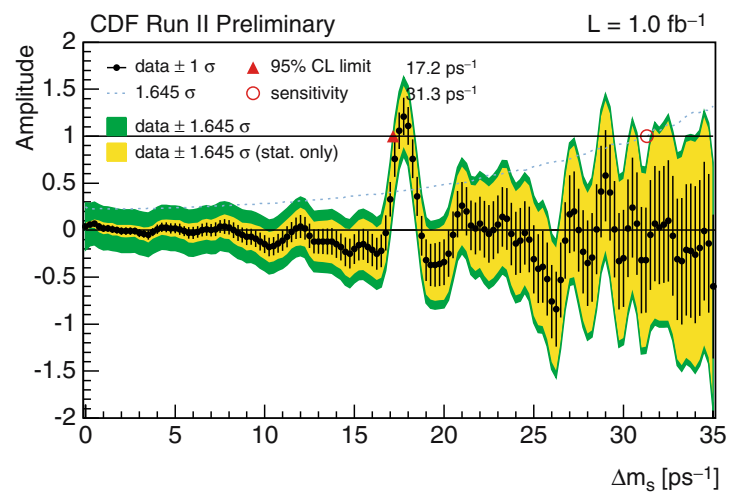

Fig. 11. Observation of large $B_{s}$ mixing with amplitude scan method (from [24]). 
Besides reconstruction in semileptonic modes like $B_{s} \rightarrow D_{s} \ell \nu$, CDF has the advantage of reconstruction in hadronic modes such as $D_{s} \pi$, which has better proper time resolution $(25 \mu \mathrm{m})$ than in semileptonic mode. Furthermore, besides opposite side tagging (similar to B factories), CDF enjoys same side tagging of the $B_{s}$ flavor by the associated $K^{ \pm}$, improving the overall effectiveness, $\varepsilon D^{2}$, where $\varepsilon$ is efficiency and $D=1-2 w$ is dilution.

The measured $\Delta m_{B_{s}}$ is consistent with SM, but it can be said that it is slightly on the low side, when compared to predictions made beforehand. With the measured $\Delta m_{B_{d}}$, one can extract

$$
\left|\frac{V_{t d}}{V_{t s}}\right|=\xi \sqrt{\frac{\Delta m_{B_{d}}}{\Delta m_{B_{s}}} \frac{m_{B_{s}}}{m_{B_{d}}}} \simeq 0.21,
$$

where $\xi=\sqrt{f_{B_{s}}^{2} B_{B_{s}} / f_{B_{d}}^{2} B_{B_{d}}}$ is a hadronic parameter ratio that has to be calculated, e.g., by lattice methods, which dominates the error on $\left|V_{t d} / V_{t s}\right|$.

The question of interest is now the size of CPV in $B_{s}$ system. Tevatron Run II would end by 2009, with up to $8 \mathrm{fb}^{-1}$ expected per experiment, while LHCb will start running with LHC commissioning in 2008. LHCb should be able to repeat the "CDF measurement" of $\Delta m_{B_{s}}$ in an "instant", and should be able to probe $\sin 2 \phi_{B_{s}}$ at the SM level of $-4 \%$. However, if $\left|\sin 2 \phi_{B_{s}}\right|$ is large, say $0.5-0.7$, perhaps the Tevatron could draw another punch?

\section{$b \rightarrow d \gamma$ and $B \rightarrow K \bar{K}$}

Another probe of $\left|V_{t d} / V_{t s}\right|$ is $b \rightarrow d \gamma . B \rightarrow K^{*} \gamma$ was already established in 1993, while $B \rightarrow \rho \gamma$ appeared at the B factories in 2006. At the moment there is still some conflict between Belle and BaBar on the actual strength. At the same time, the gluonic $b \rightarrow d \bar{s} s$ penguin processes (changing $\bar{s}$ to $\bar{d}$, and the $u \bar{u}$ pair from gluon to $s \bar{s}) B \rightarrow \bar{K}^{0} K^{0}, \bar{K}^{0} K^{+}$have also been established by Belle and BaBar. However, the precision for $\left|V_{t d} / V_{t s}\right|$ determination is not competitive with $\Delta m_{B_{d}} / \Delta m_{B_{s}}$.

\section{The Two "Fitters"}

Two competing groups [21] have formed that try to combine all data and all theory approaches and uncertainties, and display the information on the unitarity triangle of Fig. 2 (Eq. (5)). The results are displayed in Fig. 12. We shall not go into the details, nor into the debate of "frequentist" or "Bayesian" approach. At the core, one can see the unitarity triangle. At worst, these figures are some form of "Modern Art".

\subsection{Large Missing Energy Decays: $B \rightarrow \tau \nu$ etc.}

In discussing $B_{s}$ mixing and $V_{t d} / V_{t s}$, we mentioned hadronic parameter dependence such as on $f_{B_{q}}$, the meson decay constant. The $B^{ \pm} \rightarrow \tau^{ \pm} \nu$ decay, 

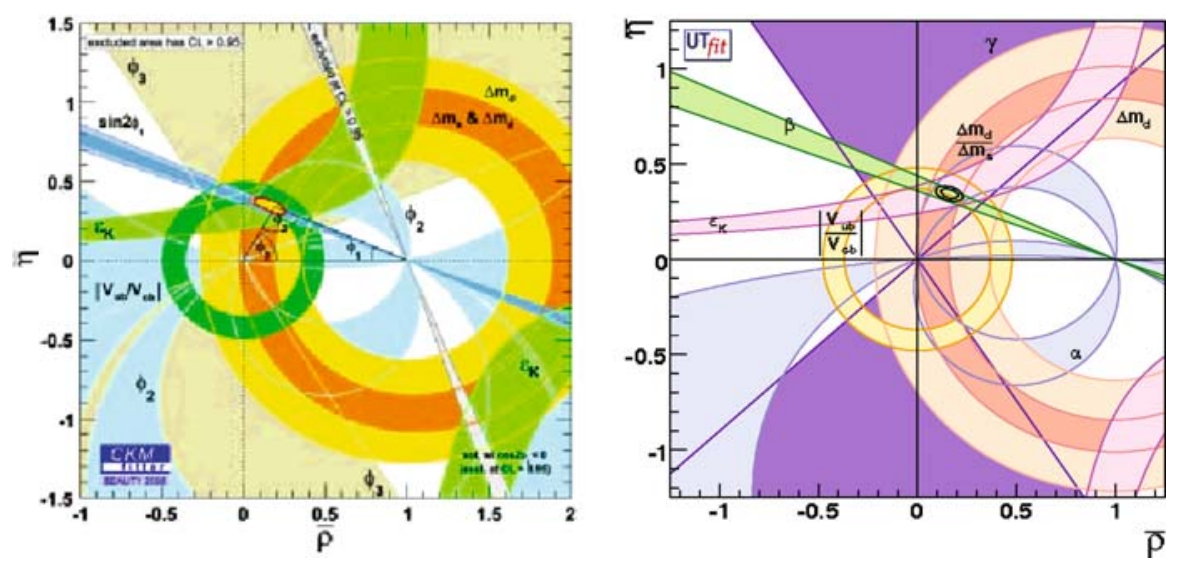

Fig. 12. The CKMfitter and UTfit representation of current information on the unitarity triangle of Eq. (5) (from relevant web pages [21]).

entirely analogous to $\pi^{ \pm} \rightarrow \mu^{ \pm} \nu$ and $K^{ \pm} \rightarrow \mu^{ \pm} \nu$, probes the $B$ meson decay constant directly. The process goes through an annihilation diagram as in Fig. 3(c), where the $b$ and $\bar{u}$ quarks annihilate into a $W^{-}$boson, which converts into $\tau^{-} \nu$. The annihilation probes the wave function at the origin, where the meson decay constant is a measure.

The formula for $B^{ \pm} \rightarrow \tau^{ \pm} \nu$ decay is easily related to muon decay, except one now has only two body phase space. We have

$$
\Gamma(B \rightarrow \tau \nu)=\frac{G_{F}^{2}}{8 \pi} m_{B} m_{\tau}^{2}\left(1-\frac{m_{\tau}^{2}}{m_{B}^{2}}\right) f_{B}^{2}\left|V_{u b}\right|^{2},
$$

which depends only on the product $f_{B}\left|V_{u b}\right|$. All hadronic dependence is absorbed into $f_{B}$, which, if computed from lattice, then $\left|V_{u b}\right|$ can be inferred from the measured rate. The nominal SM expectation is around $10^{-4}$. A measurement of $\mathcal{B}(B \rightarrow \tau \nu)$ also probes New Physics, especially the effect of a charged Higgs $H^{+}$boson, by simply modifying Eq. (29) by a factor [25]

$$
\mathcal{B}(B \rightarrow \tau \nu)=\mathcal{B}_{B \rightarrow \tau \nu}^{\mathrm{SM}} \times r_{H}, \quad r_{H}=\left(1-\frac{m_{B}^{2}}{m_{H^{+}}^{2}} \tan ^{2} \beta\right)^{2},
$$

where $\tan \beta$ is the usual ratio of vacuum expectations in models with two Higgs doublets (2HDM), such as in supersymmetry. The enhancement factor appears because of coupling to $m_{b}$ and $m_{\tau}$ in the SUSY type of 2HDM.

The challenge for measuring $B \rightarrow \tau \nu$ is as follows. Neutrino detection techniques have been developed when there is only one neutrino. Since $\tau$ decay always involve a tau neutrino, the event has at least two neutrinos, hence has both large missing energy and missing mass. However, since $B$ mesons decay semileptonically for over $20 \%$ of the time, if one misses any particle (track) 
from the other $B$ meson in the $B \bar{B}$ event, it would become background for $B \rightarrow \tau \nu$. This was difficult enough in the CLEO era when luminosity was lower. For the high luminosity B factories, detectors are not hermetic enough. Not only one has myriads of cracks, one can see from Fig. 1 that the final focus quadrupole magnets enter inside the guts of the detector, and there is a finite opening angle for active detection.

The price to be paid was the full reconstruction of the other $B$, at the cost of $10^{-3}$ efficiency. This was pursued vigorously once Belle and BaBar each accumulated several hundred million $B \bar{B}$ pairs. Finally, in 2006 Belle announced $3.5 \sigma$ evidence [26] for $B \rightarrow \tau \nu$, with $\tau \rightarrow e, \mu, \pi, \rho$ plus neutrino(s),

$$
\mathcal{B}(B \rightarrow \tau \nu)=\left(1.79_{-0.49-0.46}^{+0.56+0.39}\right) \times 10^{-4}, \quad \text { (Belle 2006) }
$$

with BaBar value lower and still lack of evidence [19]. The combined result is lower than Belle's first evidence, which is consistent with SM expectation. If one treats $f_{B}$ and $\left|V_{u b}\right|$ as known, one gets a range for $r_{H}$, which constrains the $m_{H^{+}}-\tan \beta$ plane, and can be improved in the future.

Together with $b \rightarrow s \gamma, B$ physics provides powerful constraints on New Physics such as $H^{+}$bosons.

\section{$B \rightarrow K^{(*)} \nu \nu$ or $K^{(*)}+$ Nothing}

Full reconstruction of one $B$ opens up the study of other interesting channels. The $B \rightarrow K^{(*)} \nu \nu$ decay is rather similar to $B \rightarrow \tau \nu \rightarrow \pi(\rho) \nu \nu$ in final state selection: $K(\pi)$ in place of $\pi(\pi)$. Thus, it has been searched for concurrently.

In $\mathrm{SM}$, the $B \rightarrow K^{(*)} \nu \nu$ process is induced by [17] bs $Z$ penguin and box diagrams, analogous to the $B \rightarrow K^{(*)} \ell^{+} \ell^{-}$process (which has the additional $b s \gamma$ penguin contribution). The expectation is at the $10^{-5}$ level. However, the experimental signature is just $B \rightarrow K^{(*)}+$ nothing. This nothing could also be light dark matter. It is interesting to note that, although the DAMA hint for dark matter in our solar neighborhood seems ruled out by the CDMS experiment, direct dark matter searches are not sensitive to $M<10 \mathrm{GeV}$. Thus, $B \rightarrow K^{(*)} \nu \nu$ study provides a window on dark matter search.

The experimental technique is rather similar to $B \rightarrow \tau \nu$ study: full reconstruction of "tag side" and $K+$ nothing on the signal side, Belle has searched with $535 \mathrm{M} B \bar{B}$ pairs and reported preliminary results. The upper limits reached is still 10 times the SM expectation. Put in different way, there is still room for discovery. But progress would require a Super B factory.

Also motivated by dark matter search, Belle has made specific runs on the $\Upsilon(3 S)$ to search for $\Upsilon \rightarrow$ "nothing" decays, via $\Upsilon(3 S) \rightarrow \pi^{+} \pi^{-} \Upsilon$ using $\pi^{+} \pi^{-}$ as tag. No signal was found [27], but it illustrates the prowess and versatility of B factories. The special data taking took just a few days. 


\subsection{Some Tau/Charm Topics}

\section{$\tau \rightarrow \ell \gamma$}

Radiative $\tau \rightarrow \ell \gamma$ decays are of great interest because it is extremely suppressed by the tiny neutrino mass within SM context. Analogous to the $b \rightarrow s \gamma$ transition, in supersymmetric models, for example, sneutrino-chargino or slepton-neutralino loops could induce the transition, and whether $\tau \rightarrow e \gamma$ or $\tau \rightarrow \mu \gamma$ is more important is model dependent, the branching fraction typically scaling as $\tan ^{2} \beta / M_{\text {SUSY }}^{4}$. As B factories are also $\tau$ factories, current limits have reached the $10^{-8}$ level, constraining the $\tan \beta-M_{\mathrm{SUSY}}$ plane. This complements the $\mu \rightarrow e \gamma$ and muon $g-2$ studies.

Many other lepton number (even baryon number) violating studies have been pursued.

\section{$D^{0}-\bar{D}^{0}$ Mixing}

The $K^{0}-\bar{K}^{0}$ mixing phenomenon is repeated in the $B_{d}$ and $B_{s}$ systems. The only other similar meson is the $D^{0}$, composed of $c \bar{u}$. For this system, $x_{D} \equiv$ $\Delta m_{D} / \Gamma_{D}$ is highly suppressed within SM. Unlike $K$ and $B$ mesons, $\Gamma_{D}$ has no CKM suppression. On the other hand, $\Delta m_{D}$ is highly suppressed by the GIM mechanism: the $d$ and $s$ quarks are almost degenerate on the weak scale, and their effects very effectively cancel between $V_{u d} V_{c d}^{*} \cong-V_{u s} V_{c s}^{*} \cong-\lambda$, while for $b$ quark in the box diagram, the CKM factor $\left|\left(V_{u b} V_{c b}^{*}\right)^{2}\right|<\lambda^{10}$ is highly suppressed. Thus, one expects $x_{D}^{\mathrm{SM}}$ to be very small, hence a good probe of New Physics if $x_{D}$ is dominated by short distance effects. However, SU(3) violation in $D^{0}$ and $\bar{D}^{0}$ decay to 2 - and 4-body common final states could generate $\Delta \Gamma_{D}$, or mixing in rate.

After having a hint for some time, both Belle and BaBar have now found evidence pointing to $y_{D} \equiv \Delta \Gamma_{D} / 2 \Gamma_{D}$. Belle finds evidence [28] for $y_{C P}(=y$ if CPV small), by comparing $D^{0}$ lifetimes in $K^{+} K^{-}, \pi^{+} \pi^{-}$channels vs $K^{+} \pi^{-}$. BaBar finds evidence [29] for $y^{\prime}$ in $D^{0} \rightarrow K^{+} \pi^{-}$decay, but together with a negative $x^{\prime 2}$ with a large correlation between the two. Taken together, these results imply that $y_{D}$ seems to be at the $\%$ level.

Having $y_{D} \sim \%$ level could generate $x_{D}$ at similar level [30]. But this would be long distance, $\mathrm{SU}(3)$ breaking physics, and less interesting. The real interest is in $x_{D}$ generated by short distance effect, i.e. from New Physics. It seems that, again, CPV would be the path to pursue, but this would require much higher luminosities as there is no indication of it so far.

$$
D_{q}^{+} \rightarrow \ell^{+} \nu \text { and } f_{D_{q}}
$$

Whether short or long distance effect, the $D$ meson decay constant $f_{D}$ enters decay and mixing amplitudes. Furthermore, comparing between experiment 
and lattice results for $f_{D_{q}}$ provides an important crosscheck of lattice calculations for $f_{B}$, which is important for extracting information from $B_{d}$ and $B_{s}$ mixing, as well as $B \rightarrow \tau \nu$. Thus, measurement of $f_{D_{q}}$ is of great interest.

The rate $\Gamma\left(D_{q}^{+} \rightarrow \ell^{+} \nu\right)$ is of the form of Eq. (29) by trivial replacement. Unfortunately, because there is no $\tan \beta$ enhancement of the charged Higgs coupling to charm, while the $d$ and $s$ quark masses are too light, there is no sensitivity to $H^{+}$like in $B \rightarrow \tau \nu$. The current experimental value for $f_{D_{s}}$ is of order $280 \mathrm{MeV}$, while $f_{D^{+}}$is of order $220 \mathrm{MeV}$, which are higher than lattice results, but the ratio $f_{D_{s}} / f_{D^{+}} \sim 1.26$ is in better agreement with lattice.

\section{Spectroscopy — Spectacular Renaissance!}

The discovery of new charmed-strange $\left(D_{s J}[3]\right)$ and charmonium $(X(3872)$ [2]) mesons have lead to a renaissance, after a 25 year hiatus, in meson spectroscopy, which we cannot do justice here, because of space, and because we are concerned with flavor rather than aspects of strong interactions.

There are now new $D_{s J}$ mesons decaying to $D K$, new baryons decaying to $D p$, new charmed baryons decaying to $\Lambda_{c} K \pi$, etc., and there is no good interpretations yet. On the charmonium side, besides the narrow $X(3872)$ found by Belle, a broad $1^{--} Y(4260)$ state was found by BaBar. Both states decay to $\pi^{+} \pi^{-} J / \psi$. A proliferation of states has followed: $X(3940), Y(3940)$, $Y(4350)$, etc. While it is not yet clear how to place these states, the future is clearly wide open.

\section{Future Perspective}

Flavor physics is reaching a new juncture, and will undergo a major change after 2008. Some facilities would be closing down, some would experience rebirth or transformation. And, there will be the new kid in town: LHCb. One should also take the broader view and, together with the other two subjects of electroweak symmetry breaking and mass generation, neutrino physics and lepton sector, see flavor physics within the common quest to unravel New Physics.

\section{Closings}

With the Linac used as part of the new LCLS light source, PEP-II and BaBar will stop in 2008. The CLEO-c detector, morphed from CLEO-III by pressure of the B factories, would also end after taking some $\psi(3770) \rightarrow D \bar{D}$ data.

With LHC physics run to start in 2008, the Tevatron would also close down around that time. But Tevatron B physics would continue to deliver good results in $\mathcal{A}_{K^{-} \pi^{+}}$, could pull a coup in CPV in $B_{s} \rightarrow J / \psi \phi$, and could make other discoveries such as DCPV in $B^{-} \rightarrow J / \psi K^{-}$, as well as "owning" heavy $b$ hadrons until LHC turn-on. 


\section{Rebirth}

With CLEO-c moving into its ground, BEPC and BES was pushed to upgrade. With two rings, BEPC-II is designed to reach 10 times the luminosity at CLEO-c, and commissioning will start in 2007. The detector also undergoes a major upgrade to BES-III, aimed for the physics of charm and charm spectroscopy starting in 2008.

As the brightest collider in the world, and with superb physics output, KEKB and Belle aim for a well-deserved major upgrade to a Super B factory, with increase of luminosity by two orders of magnitude. A plan ("Grand Lepton Collider Project") has been worked out with KEK to integrate and optimize accelerator manpower usage between SuperKEKB and ILC (as well as J-PARC), where work on SuperKEKB can be viewed as an early phase of ILC accelerator manpower buildup. The problem is a budget shortfall in maintaining such an ambitious program, especially during 2008-2010 when KEK also has to run the J-PARC proton facility which is just being finished. Hopefully, with the success of the Japanese particle physics program, and the overall growth of Asia, the ambitious program can be carried through.

While one can be optimistic for SuperKEKB, there is another "Linear Collider"-inspired SuperB proposal at INFN, Italy. With low emittance, low beta and low current, the novel design is quite different from the more traditional high current, high beam-beam parameter SuperKEKB.

\section{Newborn}

With "regeneration" pains on the $e^{+} e^{-}$front, hadronic collider B facilities are entering a new phase: LHCb. With the capabilities of B studies in a hadronic environment demonstrated at the Tevatron, LHCb capitalizes on the high $b$ cross section and optimize to a forward detector design, as well as choice of luminosity. It has efficient trigger for hadronic and leptonic modes, excellent tracking and vertexing, and two RICH (Ring Imaging Cherenkov) detectors for excellent PID. What LHCb is somewhat weaker on is photons, or ECAL.

\section{LHCb vs Super B}

So, the future of flavor physics seems to be LHCb vs a Super B factory. In fact, they are quite complementary.

With much larger production cross section and the large boost, together with triggering, vertexing and PID capabilities, LHCb can do very well in most processes not involving $\gamma$ (hence $\pi^{0}$ ) or neutrinos, and if there are not too many tracks. In particular, important topics of $B_{s}$ system, such as TCPV, $B_{s} \rightarrow \mu^{+} \mu^{-}$, and especially heavy $b$ hadron spectroscopy, would be the domain of LHCb. Although B factories can run on the $\Upsilon(5 S)$ to study $B_{s}$ physics, it would not be easy to compete with LHCb and exclusively run at that energy. On traditional programs such as $\phi_{1} / \beta, \phi_{2} / \alpha, \phi_{3} / \gamma$ for $B_{d}$ and $B_{u}$ systems, 
and DCPV in inclusive $b \rightarrow s \gamma$, the two approaches are complementary. When there is more than one photon, or on subtleties such as the lack of knowledge of the $B$ direction in hadronic production, Super B would prevail. Examples are $\mathcal{S}_{\eta^{\prime} K_{S}}$, and especially $\mathcal{S}_{K_{S} \pi^{0} \gamma}$, where Super B has the advantage of knowing the $B$ direction (basically the boost direction) for forming the " $K_{S}$ " vertex. When it comes to processes involving neutrinos, such as $B \rightarrow K^{(*)} \nu \nu, \tau \nu, D^{(*)} \tau \nu$ etc., then $\mathrm{LHCb}$ is powerless. This also holds for inclusive processes, where the clean $e^{+} e^{-}$environment and the exclusive $B \bar{B}$ final state, are great advantages. Similarly, the clean environment gives Super B an edge on tau/charm topics such as $\tau \rightarrow \mu \gamma$ and $D^{0}-\bar{D}^{0}$ mixing.

An observation illustrates the point: the first few years of running of $\mathrm{LHCb}$ would not drastically improve the significance of the $\Delta \mathcal{S}$ problem. LHCb could approach $\mathcal{S}_{\phi K_{S}}$, but not very well, because of the relatively long-lived nature of $K_{S} \rightarrow \pi^{+} \pi^{-}$, while $\phi \rightarrow K^{+} K^{-}$is too close to threshold, so the two kaon tracks do not separate well at production. For $K_{S} \pi^{0}$, again one cannot find a vertex, while for $\eta^{\prime} K_{S}$, reconstruction of $\eta^{\prime}$ is not so easy, and one still has the $K_{S}$ to deal with. Thus, improved understanding of whether there is a genuine problem in $\Delta \mathcal{S}$ requires a Super B factory. It remains to be seen how well can LHCb do with DCPV involving $\pi^{0} \mathrm{~S}$ in final state, such as $\mathcal{A}_{B^{+} \rightarrow K^{+} \pi^{0}}$. For these issues, and other more practical ones, the LHCb experiment is already discussing upgrade possibilities to improve detector performance.

To say the least, the superb hadronic collider approach of LHCb would greatly enrich flavor studies, especially in $B_{s}$ system. But a Super B factory would complement the program and make it more complete. This would remain true for the next decade or two.

\section{The Broad Perspective}

The flavor program should be viewed in the broader perspective of our search for physics beyond SM, i.e. New Physics.

The high energy frontier will open up greatly with the LHC, which has as its main goal the search for the Higgs boson, to understand electroweak symmetry breaking and the origins of mass, where SUSY or other phenomena such as large extra dimensions may be uncovered. If LHC uncovers new particles, the study of virtual effects, and the effects on $B, D, \tau$ flavor, as well as kaon and muon sectors, would be of common interest.

It can be said that New Physics has already been seen in the neutrino sector: neutrinos have mass [1]. Thus, the neutrino sector itself has a CKMlike mixing and CPV program to be explored, and one current focus is to try to measure $\sin 2 \theta_{13}$, since the nonvanishing of which is needed for the continued CPV search. Neutrino mass also touches on many subjects, from cosmology, to lepton flavor violation. These become common cause not only with the Super B factory program of lepton flavor and $C P$ violation in $\tau$ sector, such as $\tau \rightarrow \ell \gamma$, but also the $\mu \rightarrow e \gamma$ and muon $g-2$ programs. 


\section{Conclusion}

We have covered the current activities on the flavor and B physics front, from the Standard Model predictions, to potential New Physics indications. The $\mathrm{B}$ factories have performed spectacularly, as we have covered in the main part of CPV studies of $\phi_{1}, \phi_{2}$ and $\phi_{3}$. The main goal of the B factories, the measurement of $\sin 2 \phi_{1}$, has already become a precision measurement!

All of us are of course more interested in uncovering New Physics, as implied by the title of this School. The detailed study of SM predictions, such as overconstraining the CKM unitarity triangle, is a more conservative and painstaking approach. The other, more frontal kind of approach is illustrated by the $\Delta \mathcal{S}$ problem, where experimental measurement suggests that it may be negative, while theoretical modelling suggests the opposite. Alas, the hint is only at $2.6 \sigma$, and cannot be drastically improved for the next few years.

We have advocated a second possible hint for New Physics, in the difference of $\Delta \mathcal{A}_{K \pi}=\mathcal{A}_{B^{+} \rightarrow K^{+} \pi^{0}}-\mathcal{A}_{B^{0} \rightarrow K^{+} \pi^{-}} \neq 0$. This can indeed be accounted for partially with an enhanced color-suppressed amplitude $C$, but the enhancement needed would be very great indeed, especially if one also wishes to touch the $\Delta \mathcal{S}$ problem. In fact $\Delta \mathcal{A}_{K \pi}$ is a window on New Physics from the electroweak or $Z$ penguin: the virtual $Z$ converts to $\pi^{0}$ but not $\pi^{-}$, hence affecting $\Delta \mathcal{A}_{K \pi}$, and probes New Physics CPV in the $b s Z$ coupling. Since the box diagram and the $Z$ penguin are closely related, from this perspective I expect interesting TCPV effects could emerge in $B_{s}$ mixing as well as $D^{0}$ mixing, and maybe in DCPV in $B^{+} \rightarrow J / \psi K^{+}$.

It is hard to foresee where New Physics would first emerge. The LHC is a good bet, but the LHC could also just uncover SM Higgs (or not even that!), and we could be left with flavor violation studies as our best probe for the New Physics landscape. Alternatively, it is not impossible that the Tevatron could uncover evidence for large TCPV in $B_{s}$ system, such as $\left|\sin 2 \Phi_{B_{s}}\right| \sim 0.5$ or higher, before LHC turn on. New Physics involving flavor would come to the forefront, and the energy frontier opened by LHC would explore it further.

We are entering a potentially very exciting era.

\section{Acknowledgements}

I sincerely wish to thank Meltem Serin-Zeyrek, as well as the co-organizers and all the supporting workers of ISSMB06, which made the meeting at Akyaka such an enjoyable one. My only regret is that I did not have time to stay long enough, hence I look forward to a more leisurely return in the future. Hopefully the School becomes a regular one. I have enjoyed Belle very much as a collaboration, where some of the figures have been used freely. This work is supported by the National Science Council and the Ministry of Education of Taiwan. 


\section{References}

1. H.T. Wong, this volume.

2. S.K. Choi et al. [Belle Collab.], Phys. Rev. Lett. 91, 262001 (2003).

3. B. Aubert et al. [BaBar Collab.], Phys. Rev. Lett. 90, 242001 (2003).

4. E. Fernandez et al. [MAC Collab.], Phys. Rev. Lett. 51, 1022 (1983); N.S. Lockyer et al. [MARK II Collab.], ibid. 51, 1316 (1983).

5. S. Stone, plenary talk at Lepton-Photon Symposium, Cornell, USA, 1983.

6. W.M. Yao et al. [Particle Data Group], J. Phys. G 33, 1 (2006).

7. H. Albrecht et al. [ARGUS Collab.], Phys. Lett. B 192, 245 (1987).

8. R. Ammar et al. [CLEO Collab.], Phys. Rev. Lett. 71, 674 (1993); M.S. Alam et al. [CLEO Collab.], ibid. 74, 2885 (1995).

9. S. Bertolini, F. Borzumati and A. Masiero, Phys. Rev. Lett. 59, 180 (1987); N.G. Deshpande et al., ibid. 59, 183 (1987).

10. B. Grinstein and M.B. Wise, Phys. Lett. B 201, 274 (1988); W.S. Hou and R.S. Willey, ibid. B 202, 591 (1988).

11. Attributed to P. Oddone, 1987.

12. F. Abe et al. [CDF Collab.], Phys. Rev. Lett. 68, 3403 (1992).

13. A. Go et al. [Belle Collab.], quant-ph/0702267.

14. B. Aubert et al. [BaBar Collab.], Phys. Rev. Lett. 93, 131801 (2004); Y. Chao et al. [Belle Collab.], ibid. 93, 191802 (2004).

15. R. Godang et al. [CLEO Collab.], Phys. Rev. Lett. 80, 3456 (1998).

16. B.H. Behrens et al. [CLEO Collab.], Phys. Rev. Lett. 80, 3710 (1998); T.E. Browder et al. [CLEO Collab.], ibid. 81, 1786 (1998).

17. W.S. Hou, R.S. Willey and A. Soni, Phys. Rev. Lett. 58, 1608 (1987).

18. K. Abe et al. [Belle Collab.], Phys. Rev. Lett. 88, 021801 (2002); J. Kaneko et al. [Belle Collab.], ibid. 90, 021801 (2003).

19. The latest result, including many measurements of rare decays, can be found at the HFAG (Heavy Flavor Averaging Group) webpage.

20. A.B. Carter and A.I. Sanda, Phys. Rev. Lett. 45, 952 (1980).

21. See the webpages of http://www.slac.stanford.edu/xorg/ckmfitter/, and http://utfit.roma1.infn.it/.

22. N. Isgur and M.B. Wise, Phys. Lett. B 232, 113 (1989).

23. A. Giri, Y. Grossman, A. Soffer and J. Zupan, Phys. Rev. D 68, 054018 (2003);

A. Bondar, 2002 (unpublished).

24. A. Abulencia et al. [CDF Collab.], Phys. Rev. Lett. 97, 242003 (2006).

25. W.S. Hou, Phys. Rev. D 48, 2342 (1993).

26. K. Ikado et al. [Belle Collab.], Phys. Rev. Lett. 97, 251802 (2006).

27. O. Tajima et al. [Belle Collab.], Phys. Rev. Lett. 98, 132001 (2007).

28. M. Staric et al. [Belle Collab.], Phys. Rev. Lett. 98, 211803 (2007).

29. B. Aubert et al. [BaBar Collab.], Phys. Rev. Lett. 98, 211802 (2007).

30. A.F. Falk et al., Phys. Rev. D 69, 114021 (2004). 